\title{
Questionable Claims Found in Ramanujan's Lost Notebook
}

\author{
BRUCE C. BERNDT ${ }^{1}$, O-YEAT CHAN, SUNG-GEUN LIM ${ }^{2}$, and ALEXANDRU ZAHARESCU ${ }^{3}$
}

\section{Introduction}

Recall that Ramanujan's lost notebook [27] was written in the last year of his life. We can forgive him if, in his insufferable pain, not everything he wrote down was correct. The purpose of this paper is to examine some of these questionable results. In particular, we concentrate on three "identities" found on pages 335 and 336 in [27]. At the close of this paper, we briefly mention some further questionable results from [27]. Recall also that when Ramanujan's lost notebook [27] was published in 1988, other fragments and partial manuscripts were also published with the lost notebook. The aforementioned two pages are, in fact, not from the lost notebook. Possibly, Ramanujan realized indeed that these results were speculative and had put them aside or among other papers separate from those comprising what we now call the lost notebook. We are uncertain if the formula in question on page 335 is true or false, but if the formula is not correct, we are confident that a corrected version indeed does exist. For the formulas on page 336, Ramanujan possibly possessed correct identities and used some unknown formal procedure to replace certain expressions by divergent series in order to make the identities more attractive. Therefore, we are writing this paper on questionable formulas, because we truly feel that corrected versions do indeed exist. Since we do not have these correct versions, it is difficult to claim that we have made progress toward finding them, but we hope that the ideas and partial results gathered here might provide insights to others.

As we shall see in Section 4, the formula on page 335, when (possibly) corrected, may have an application to the problem of finding the exact order of magnitude for the "error term" $\Delta(x)$ in the famous "divisor problem". If $d(n)$ denotes the number of positive divisors of the positive integer $n$, then recall that $\Delta(x)$ is defined for $x>0$ by

$$
\sum_{n \leq x}^{\prime} d(n)=x(\log x+(2 \gamma-1))+\frac{1}{4}+\Delta(x)
$$

where $\gamma$ denotes Euler's constant and the prime / on the summation sign on the left-hand side indicates that if $x$ is an integer, then only $\frac{1}{2} d(x)$ is counted. The questionable formula to which we allude is actually one of a pair of formulas stated by Ramanujan on page 335 of [27]. The other formula, which is connected with the famous "circle problem," is in fact correct and has been proved by Berndt and Zaharescu [14].

A pair of formulas also comprises page 336 in [27]. These two formulas are invalid, because each contains divergent series. Ramanujan frequently enjoyed stating identities in an unorthodox

\footnotetext{
${ }^{1}$ Research partially supported by grant MDA904-00-1-0015 from the National Security Agency.

${ }^{2}$ This work was supported by a Korea Research Foundation Grant funded by the Korean Government(MOEHRD).(KRF- 2006-214-C00003)

${ }^{3}$ Research partially supported by grant DMS-0456615 from the National Science Foundation.
} 
fashion in order to surprise or puzzle his audience. As indicated above, we timorously conjecture that Ramanujan had established correct identities in each case.

\section{Statements of the Questionable Formulas}

We begin by stating the previously established formula of the pair of results written on page 335 of [27]. Recall that the ordinary Bessel function of order $\nu$ is defined by

$$
J_{\nu}(z):=\sum_{n=0}^{\infty} \frac{(-1)^{n}}{n ! \Gamma(\nu+n+1)}\left(\frac{z}{2}\right)^{\nu+2 n}, \quad 0<|z|<\infty, \quad \nu \in \mathbb{C} .
$$

To state Ramanujan's first claim, we also need to define

$$
F(x)= \begin{cases}{[x],} & \text { if } x \text { is not an integer, } \\ x-\frac{1}{2}, & \text { if } x \text { is an integer, }\end{cases}
$$

where, as customary, $[x]$ is the greatest integer less than or equal to $x$.

Theorem 2.1. If $0<\theta<1$ and $x>0$, then

$$
\begin{aligned}
\sum_{n=1}^{\infty} F\left(\frac{x}{n}\right) \sin (2 \pi n \theta) & =\pi x\left(\frac{1}{2}-\theta\right)-\frac{1}{4} \cot (\pi \theta) \\
+ & \frac{1}{2} \sqrt{x} \sum_{m=1}^{\infty} \sum_{n=0}^{\infty}\left\{\frac{J_{1}(4 \pi \sqrt{m(n+\theta) x})}{\sqrt{m(n+\theta)}}-\frac{J_{1}(4 \pi \sqrt{m(n+1-\theta) x})}{\sqrt{m(n+1-\theta)}}\right\} .
\end{aligned}
$$

Ramanujan's formulation of (2.3) is slightly different but easily seen to be equivalent. Berndt and Zaharescu proved (2.3) with the order of summation on the right-hand side reversed [14]. Note that the series on the left-hand side of (2.3) is finite and discontinuous at integral values of $x$. To examine the right-hand side, we recall that [31, p. 199], as $x \rightarrow \infty$,

$$
J_{\nu}(x) \sim\left(\frac{2}{\pi x}\right)^{1 / 2} \cos \left(x-\frac{1}{2} \nu \pi-\frac{1}{4} \pi\right) .
$$

Hence, as $m, n \rightarrow \infty$, the terms of the double series on the right-hand side of (2.3) are asymptotically equal to

$$
\frac{1}{\pi \sqrt{2} x^{1 / 4} m^{3 / 4}}\left(\frac{\cos \left(4 \pi \sqrt{m(n+\theta) x}-\frac{3}{4} \pi\right)}{(n+\theta)^{3 / 4}}-\frac{\cos \left(4 \pi \sqrt{m(n+1-\theta) x}-\frac{3}{4} \pi\right)}{(n+1-\theta)^{3 / 4}}\right) .
$$

Theorem 2.1 is related to the "circle problem." Let $r_{2}(n)$ denote the number of representations of the positive integer $n$ as a sum of two squares. Recall that the famous circle problem is to determine the precise order of magnitude for the "error term" $P(x)$ defined by

$$
\sum_{0 \leq n \leq x}{ }^{\prime} r_{2}(n)=\pi x+P(x)
$$

where the prime $/$ on the summation sign on the left side indicates that if $x$ is an integer, only $\frac{1}{2} r_{2}(x)$ is counted. In connection with his work on the circle problem, in 1915, Hardy [19], [21, pp. 243-263] proved that

$$
\sum_{0 \leq n \leq x}{ }^{\prime} r_{2}(n)=\pi x+\sum_{n=1}^{\infty} r_{2}(n)\left(\frac{x}{n}\right)^{1 / 2} J_{1}(2 \pi \sqrt{n x}) .
$$

Observe that the summands in the series on the right side of (2.7) are similar to those on the right side of (2.3). Berndt and Zaharescu [14] employed Theorem 2.1 to prove a general representation 
for weighted divisor sums. A corollary of their result is the representation

$$
\sum_{0 \leq n \leq x}{ }^{\prime} r_{2}(n)=\pi x+2 \sqrt{x} \sum_{n=0}^{\infty} \sum_{m=1}^{\infty}\left\{\frac{J_{1}\left(4 \pi \sqrt{m\left(n+\frac{1}{4}\right) x}\right)}{\sqrt{m\left(n+\frac{1}{4}\right)}}-\frac{J_{1}\left(4 \pi \sqrt{m\left(n+\frac{3}{4}\right) x}\right)}{\sqrt{m\left(n+\frac{3}{4}\right)}}\right\} .
$$

It is not difficult to show the equivalence of (2.7) and (2.8); this equivalence will be demonstrated in a paper by Berndt and Zaharescu under preparation.

We now state Ramanujan's companion to (2.3). Let $Y_{\nu}(z)$ be the second solution of Bessel's differential equation of order $\nu$, usually so denoted, and let $K_{\nu}(z)$ denote the modified Bessel function of order $\nu$, usually so denoted. Following Ramanujan, we introduce the notation

$$
I_{\nu}(z):=-Y_{\nu}(z)-\frac{2}{\pi} K_{\nu}(z)
$$

ENTRY 2.2. Let $F(x)$ be defined by (2.2). For $x>0$ and $0<\theta<1$,

$$
\begin{aligned}
\sum_{n=1}^{\infty} F\left(\frac{x}{n}\right) \cos (2 \pi n \theta) & =\frac{1}{4}-x \log (2 \sin (\pi \theta)) \\
+ & \frac{1}{2} \sqrt{x} \sum_{m=1}^{\infty} \sum_{n=0}^{\infty}\left\{\frac{I_{1}(4 \pi \sqrt{m(n+\theta) x})}{\sqrt{m(n+\theta)}}+\frac{I_{1}(4 \pi \sqrt{m(n+1-\theta) x})}{\sqrt{m(n+1-\theta)}}\right\} .
\end{aligned}
$$

From standard asymptotic formulas for Bessel functions [31, pp. 199, 202],

$$
\begin{aligned}
Y_{\nu}(x) & =\sqrt{\frac{2}{\pi x}} \sin \left(x-\frac{\pi \nu}{4}-\frac{\pi}{4}\right)+O\left(\frac{1}{x^{3 / 2}}\right), \\
K_{\nu}(x) & =\sqrt{\frac{\pi}{2 x}} e^{-x}+O\left(e^{-x} \frac{1}{x^{3 / 2}}\right),
\end{aligned}
$$

as $x \rightarrow \infty$. Thus, we see that the summands in (2.10) have the same sort of asymptotic behavior as those in (2.3), as exhibited in (2.5). However, in the summands of (2.3), the pairs of Bessel functions on the right-hand side are separated by minus signs, while the corresponding pairs of Bessel functions in (2.10) are separated by plus signs.

After the statement of Theorem 2.1, we remarked that (2.3) was first proved with the order of summation on the right-hand side reversed. Unfortunately, this method fails when attempting to prove Entry 2.2. In particular, the required integrals diverge.

The right-hand side of (2.10) is reminiscent of Voronoï's [30] famous formula

$$
\sum_{n \leq x}^{\prime} d(n)=x(\log x+(2 \gamma-1))+\frac{1}{4}+\sum_{n=1}^{\infty} d(n)\left(\frac{x}{n}\right)^{1 / 2} I_{1}(4 \pi \sqrt{n x}),
$$

where $x>0$ and $I_{1}(z)$ is defined by (2.9). However, in contrast to our derivation of (2.8) from (2.3) (with the order of summation reversed), we are unable to derive (2.13) from (2.10), if indeed (2.10) is true. However, in Section 4, we prove that if (2.10) is true, then a limiting case of it along with (2.13) establishes another identity for Bessel function series.

In the next section, we provide an attempt to prove (2.10). Although our efforts are unsuccessful, our arguments indicate that possibly (2.10) is incomplete, i.e., there are some missing expressions in (2.10). However, we are unable to identify these missing terms (if indeed there are missing terms). In Section 5, we discuss numerical calculations that are inconclusive about the truth of either Entry 2.2 or its alternative version with minus signs in the summands separating the Bessel functions on the right-hand side. Moreover, our calculations on the convergence of the double series do not convince us that the series indeed does converge.

The two formulas on page 335 are clearly motivated by the circle and divisor problems. Note that Hardy's two papers [19] and [20] on the circle and divisor problems, respectively, were written in 1915 and 1916, respectively, years in which Ramanujan resided in Cambridge. 
In [19], Hardy related a beautiful identity of Ramanujan connected with $r_{2}(n)$, namely, for $a, b>0,[\mathbf{1 9}$, p. 283], [21, p. 263],

$$
\sum_{n=0}^{\infty} \frac{r_{2}(n)}{\sqrt{n+a}} e^{-2 \pi \sqrt{(n+a) b}}=\sum_{n=0}^{\infty} \frac{r_{2}(n)}{\sqrt{n+b}} e^{-2 \pi \sqrt{(n+b) a}},
$$

which is not given elsewhere in any of Ramanujan's published or unpublished work, and which is further evidence that Ramanujan and Hardy probably discussed the circle problem and likely the divisor problem as well. In [19], [21, pp. 243-263], Hardy proved that

$$
P(x)=\Omega\left((x \log x)^{1 / 4}\right),
$$

i.e., for every positive constant $A$, there exists a sequence (depending on $A$ ) $\left\{x_{n}\right\}, n \geq 1$, tending to $\infty$ such that $\left|P\left(x_{n}\right)\right|>A\left(x_{n} \log x_{n}\right)^{1 / 4}, n \geq 1$. If (2.14) is differentiated with respect to $b$, and if we then let $a$ tend to 0 , we obtain an identity which was crucial for Hardy's proof of (2.15), and which had earlier been proved by Hardy in a paper written in 1908 [18, p. 373], [22, pp. 434-452, Equation (74), p. 450].

We next present the two questionable claims on page 336 of $[\mathbf{2 7}]$.

ENTRY 2.3. Let $\sigma_{s}(n)=\sum_{d \mid n} d^{s}$, and let $\zeta(s)$ denote the Riemann zeta function. Then

$$
\begin{aligned}
\Gamma(s & \left.+\frac{1}{2}\right)\left\{\frac{\zeta(1-s)}{\left(s-\frac{1}{2}\right) x^{s-\frac{1}{2}}}+\frac{\zeta(-s) \tan \frac{1}{2} \pi s}{2 x^{s+\frac{1}{2}}}\right. \\
& \left.+\sum_{n=1}^{\infty} \frac{\sigma_{s}(n)}{2 i}\left\{(x-i n)^{-s-\frac{1}{2}}-(x+i n)^{-s-\frac{1}{2}}\right\}\right\} \\
= & (2 \pi)^{s}\left\{\frac{\zeta(1-s)}{2 \sqrt{\pi x}}-2 \pi \sqrt{\pi x} \zeta(-s) \tan \frac{1}{2} \pi s\right. \\
& \left.+\sqrt{\pi} \sum_{n=1}^{\infty} \frac{\sigma_{s}(n)}{\sqrt{n}} e^{-2 \pi \sqrt{2 n x}} \sin \left(\frac{\pi}{4}+2 \pi \sqrt{2 n x}\right)\right\} .
\end{aligned}
$$

ENTRY 2.4. Let $\sigma_{s}(n)$ and $\zeta(s)$ be as in the preceding entry. If $\alpha$ and $\beta$ are positive numbers such that $\alpha \beta=4 \pi^{2}$, then

$$
\begin{aligned}
\alpha^{(s+1) / 2}\left\{\frac{1}{\alpha} \zeta(1-s)+\right. & \left.\frac{1}{2} \zeta(-s) \tan \frac{1}{2} \pi s+\sum_{n=1}^{\infty} \sigma_{s}(n) \sin (n \alpha)\right\} \\
& =\beta^{(s+1) / 2}\left\{\frac{1}{\beta} \zeta(1-s)+\frac{1}{2} \zeta(-s) \tan \frac{1}{2} \pi s+\sum_{n=1}^{\infty} \sigma_{s}(n) \sin (n \beta)\right\}
\end{aligned}
$$

Both formulas are easily seen to be false in general, because each contains divergent series. In Sections 6 and 7, we examine these two formulas. Formula (2.17) is especially intriguing because of its beautiful symmetry, because it appears to be a relation between Eisenstein series formally extended to the real line, and because it appears to be an analogue of the Poisson summation formula or a special instance of the Voronoï summation formula.

A first examination of (2.17) reminds us of the transformation formulas for Eisenstein series, when $s$ is a positive odd integer. In [7], Berndt derived modular transformation formulas for a large class of analytic Eisenstein series. Specializing Theorem 2 of [7] for $r_{1}=r_{2}=0$ and the modular transformation $T z=-1 / z$, for $z \in \mathcal{H}=\{z: \operatorname{Im} z>0\}$, we find that, for any complex number $s$,

$$
\begin{aligned}
& z^{-s}\left(1+e^{\pi i s}\right) \sum_{n=1}^{\infty} \sigma_{s-1}(n) e^{-2 \pi i n / z}=\left(1+e^{\pi i s}\right) \sum_{n=1}^{\infty} \sigma_{s-1}(n) e^{2 \pi i n z} \\
& -z^{-s} e^{\pi i s}(2 \pi i)^{-s}\left(1+e^{\pi i s}\right) \Gamma(s) \zeta(s)+(2 \pi i)^{-s}\left(1+e^{\pi i s}\right) \Gamma(s) \zeta(s) \\
& -(2 \pi i)^{-s} \int_{C} u^{s-1} \frac{1}{e^{z u}-1} \frac{1}{e^{u}-1} d u
\end{aligned}
$$


where $\zeta(s)$ denotes the Riemann zeta function. Here $C$ is a loop beginning at $+\infty$, proceeding to the left in $\mathcal{H}$, encircling the origin in the positive direction so that $u=0$ is the only zero of $\left(e^{z u}-1\right)\left(e^{u}-1\right)$ lying "inside" the loop, and then returning to $+\infty$ in the lower half-plane. We choose the branch of $u^{s}$ with $0<\arg u<2 \pi$. Otherwise, outside the integrand, we choose the branch of $\log w$ such that $-\pi \leq \arg w<\pi$. Replacing $s$ by $s+1$ in (2.18) and slightly simplifying, we find that

$$
\begin{aligned}
& z^{-s-1} \sum_{n=1}^{\infty} \sigma_{s}(n) e^{-2 \pi i n / z}=\sum_{n=1}^{\infty} \sigma_{s}(n) e^{2 \pi i n z} \\
& +z^{-s-1} e^{\pi i s}(2 \pi i)^{-s-1} \Gamma(s+1) \zeta(s+1)+(2 \pi i)^{-s-1} \Gamma(s+1) \zeta(s+1) \\
& -\frac{(2 \pi i)^{-s-1}}{1-e^{\pi i s}} \int_{C} u^{s} \frac{1}{e^{z u}-1} \frac{1}{e^{u}-1} d u .
\end{aligned}
$$

Next, recall the functional equation of the Riemann zeta function $[\mathbf{2 9}$, p. 16, Eq. (2.1.8)]

$$
\zeta(1-s)=2^{1-s} \pi^{-s} \cos \left(\frac{1}{2} \pi s\right) \Gamma(s) \zeta(s) .
$$

If we replace $s$ by $s+1$ in (2.20), we easily see that

$$
(2 \pi i)^{-s-1} \Gamma(s+1) \zeta(s+1)=\frac{i e^{-\pi i s / 2} \zeta(-s)}{2 \sin \left(\frac{1}{2} \pi s\right)} .
$$

Using (2.21) in (2.19), we conclude that

$$
\begin{aligned}
z^{-s-1} \sum_{n=1}^{\infty} \sigma_{s}(n) e^{-2 \pi i n / z}= & \sum_{n=1}^{\infty} \sigma_{s}(n) e^{2 \pi i n z}+z^{-s-1} \frac{i e^{\pi i s / 2} \zeta(-s)}{2 \sin \left(\frac{1}{2} \pi s\right)}+\frac{i e^{-\pi i s / 2} \zeta(-s)}{2 \sin \left(\frac{1}{2} \pi s\right)} \\
& -\frac{(2 \pi i)^{-s-1}}{1-e^{\pi i s}} \int_{C} u^{s} \frac{1}{e^{z u}-1} \frac{1}{e^{u}-1} d u
\end{aligned}
$$

Omitting $n$, note that the product of the arguments in the exponentials in the two infinite series in (2.22) is equal to $4 \pi^{2}$, in accordance with the condition $\alpha \beta=4 \pi^{2}$ prescribed by Ramanujan. The identity (2.22) is the closest true identity to Entry 2.4 that we can find.

\section{A Failed Attempt to Prove Entry 2.2}

In Section 2 we emphasized that there is numerical evidence that the double series in (2.10) is possibly divergent and that perhaps Ramanujan made a mistake and that minus signs should replace the plus signs in the pairs of Bessel functions appearing on the right-hand side of (2.10), so that the formula is more like that in (2.3). In this section, we present an argument which, although involving an unjustified interchange of summation and integration, seems to provide evidence that Ramanujan did not make a mistake in signs.

Lemma 3.1. As above, let $J_{\nu}(z)$ denote the ordinary Bessel function of order $\nu$, and let $Y_{\nu}(z)$ denote the second solution of Bessel's differential equation of order $\nu$, usually so denoted. Then

$$
\begin{aligned}
\int_{0}^{\infty} \cos \left(b x^{2}\right) Y_{\nu}(c x) d x= & \frac{1}{4} \sqrt{\frac{\pi}{b}} \sec \left(\frac{\pi \nu}{2}\right)\left(\sin \left(\frac{c^{2}}{8 b}-\frac{3 \nu+1}{4} \pi\right) J_{\nu / 2}\left(\frac{c^{2}}{8 b}\right)\right. \\
& \left.+\cos \left(\frac{c^{2}}{8 b}+\frac{\nu+1}{4} \pi\right) Y_{\nu / 2}\left(\frac{c^{2}}{8 b}\right)\right),
\end{aligned}
$$

where $b, c>0$ and $-1<\operatorname{Re} \nu<1$.

Lemma 3.1 is given in $[\mathbf{1 7}$, p. 759 , formula 6.686 , no. 4]. The next lemma can be found in $[\mathbf{2 4}$, p. 360 , formula 1.16 .15 , no. 2]. However, there are three misprints in the formula. We are grateful to O. I. Marichev for verifying our corrections of the misprints. 
LEMma 3.2. Let $J_{\nu}(z)$ and $Y_{\nu}(z)$ be as in Lemma 3.1, and, as above, let $K_{\nu}(z)$ denote the modified Bessel function of order $\nu$. Then

$$
\begin{aligned}
\int_{0}^{\infty} \cos \left(b x^{2}\right) K_{\nu}(c x) d x= & \frac{\pi}{8} \sqrt{\frac{\pi}{b}} \sec \left(\frac{\pi \nu}{2}\right)\left(\cos \left(-\frac{c^{2}}{8 b}+\frac{\nu+1}{4} \pi\right) J_{\nu / 2}\left(\frac{c^{2}}{8 b}\right)\right. \\
& \left.-\sin \left(-\frac{c^{2}}{8 b}+\frac{\nu+1}{4} \pi\right) Y_{\nu / 2}\left(\frac{c^{2}}{8 b}\right)\right) .
\end{aligned}
$$

Combining Lemmas 3.1 and 3.2, we deduce the next lemma.

LEMMA 3.3. For brevity, set

$$
A=\frac{c^{2}}{8 b} .
$$

Then, under the same notation and hypotheses as in Lemmas 3.1 and 3.2,

$$
\begin{aligned}
& \int_{0}^{\infty} \cos \left(b x^{2}\right)\left\{Y_{\nu}(c x)+\frac{2}{\pi} K_{\nu}(c x)\right\} d x \\
& =\frac{1}{4} \sqrt{\frac{\pi}{b}} \sec \left(\frac{\pi \nu}{2}\right)\left(\sin \left(A-\frac{3 \nu+1}{4} \pi\right) J_{\nu / 2}(A)+\cos \left(A+\frac{\nu-1}{4} \pi\right) Y_{\nu / 2}(A)\right. \\
& \left.\quad+\cos \left(-A+\frac{\nu+1}{4} \pi\right) J_{\nu / 2}(A)-\sin \left(-A+\frac{\nu+1}{4} \pi\right) Y_{\nu / 2}(A)\right) .
\end{aligned}
$$

We now want to let $\nu \rightarrow 1^{-}$in (3.4). To do this, we need to examine the integrand in a neighborhood of the origin when $\nu=1$. First, from [17, p. 971, formula 8.444, no. 2], as $z \rightarrow 0$,

$$
Y_{1}(z)=\frac{2}{\pi} J_{1}(z)\left(\log \left(\frac{1}{2} z\right)+\gamma\right)-\frac{2}{\pi z}+O(z),
$$

where $\gamma$ denotes Euler's constant. Secondly, from [17, p. 971, formula 8.446], as $z \rightarrow 0$,

$$
\frac{2}{\pi} K_{1}(z)=\frac{2}{\pi} I_{1}(z)\left(\log \left(\frac{1}{2} z\right)+\gamma\right)+\frac{2}{\pi z}+O(z),
$$

where $I_{1}(z)$ is the modified Bessel function of order 1, usually so denoted. (This notation conflicts with that in (2.9), but we shall not return to this modified Bessel function after the present argument.) Since $J_{1}(z) \sim \frac{1}{2} z \sim I_{1}(z)$, as $z \rightarrow 0[\mathbf{1 7}$, pp. 970-971, formula 8.441, no. 2, formula 8.445], we conclude from (3.5) and (3.6) that

$$
Y_{1}(z)+\frac{2}{\pi} K_{1}(z)=\frac{2 z}{\pi}\left(\log \left(\frac{1}{2} z\right)+\gamma\right)+O(z),
$$

as $z$ tends to 0 . Thus, since $Y_{\nu}$ and $K_{\nu}$ are continuous functions of $\nu$ in a neighborhood of $\nu=1$, we can let $\nu$ tend to $1^{-}$under the integral sign on the left-hand side of (3.4). We now examine the right-hand side of (3.4). The coefficient of $Y_{\nu / 2}(A)$ on the right side is

$$
\begin{aligned}
c_{\nu}(A) & :=\cos \left(A+\frac{\nu-1}{4} \pi\right)-\sin \left(-A+\frac{\nu+1}{4} \pi\right) \\
& =\cos A\left(\cos \frac{\nu-1}{4} \pi-\sin \frac{\nu+1}{4} \pi\right)+\sin A\left(\cos \frac{\nu+1}{4} \pi-\sin \frac{\nu-1}{4} \pi\right) .
\end{aligned}
$$

It follows from an application of L'Hôspital's rule that

$$
\lim _{\nu \rightarrow 1} \frac{c_{\nu}(A)}{\cos (\pi \nu / 2)}=\sin A .
$$

The coefficient of $J_{\nu / 2}(A)$ on the right-hand side of (3.4) is

$$
\begin{aligned}
d_{\nu}(A) & :=\sin \left(A-\frac{3 \nu+1}{4} \pi\right)+\cos \left(-A+\frac{\nu+1}{4} \pi\right) \\
& =\sin A\left(\cos \frac{3 \nu+1}{4} \pi+\sin \frac{\nu+1}{4} \pi\right)+\cos A\left(\cos \frac{\nu+1}{4} \pi-\sin \frac{3 \nu+1}{4} \pi\right) .
\end{aligned}
$$


Another application of L'Hôspital's rule yields

$$
\lim _{\nu \rightarrow 1} \frac{d_{\nu}(A)}{\cos (\pi \nu / 2)}=-\cos A .
$$

In conclusion, letting $\nu$ approach $1^{-}$in (3.4), employing (3.8) and (3.9), and using the wellknown representations $[\mathbf{3 1}$, p. 64$]$

$$
J_{1 / 2}(z)=\sqrt{\frac{2}{\pi z}} \sin z \quad \text { and } \quad Y_{1 / 2}(z)=J_{-1 / 2}(z)=\sqrt{\frac{2}{\pi z}} \cos z,
$$

we deduce that

$$
\begin{aligned}
\int_{0}^{\infty} \cos \left(b x^{2}\right) & \left\{Y_{1}(c x)+\frac{2}{\pi} K_{1}(c x)\right\} d x=\frac{1}{4} \sqrt{\frac{\pi}{b}}\left(Y_{1 / 2}(A) \sin A-J_{1 / 2}(A) \cos A\right) \\
& =\frac{1}{4} \sqrt{\frac{\pi}{b}}\left(-J_{-1 / 2}(A) \sin A-J_{1 / 2}(A) \cos A\right) \\
& =\frac{1}{4} \sqrt{\frac{\pi}{b}}\left(-\sqrt{\frac{2}{\pi A}} \cos A \sin A-\sqrt{\frac{2}{\pi A}} \sin A \cos A\right) \\
& =-\frac{1}{2} \sqrt{\frac{2}{b A}} \sin A \cos A \\
& =-\frac{1}{4} \sqrt{\frac{2}{b A}} \sin (2 A) .
\end{aligned}
$$

We now return to Ramanujan's notation (2.9), so that (3.10) takes the form given below.

LEMma 3.4. With $I_{\nu}$ defined by (2.9),

$$
\int_{0}^{\infty} \cos \left(b x^{2}\right) I_{1}(c x) d x=\frac{1}{4} \sqrt{\frac{2}{b A}} \sin (2 A),
$$

where $A$ is defined by (3.3).

We need the value of one further integral. For $-1<\operatorname{Re} \nu<1$ [24, pp. 258, 343],

$$
\int_{0}^{\infty} Y_{\nu}(x) d x=-\tan \frac{\pi \nu}{2}, \quad \int_{0}^{\infty} K_{\nu}(x) d x=\frac{\pi}{2} \sec \frac{\pi \nu}{2},
$$

respectively. Hence, for $-1<\operatorname{Re} \nu<1$,

$$
\int_{0}^{\infty} I_{\nu}(x) d x=\tan \frac{\pi \nu}{2}-\sec \frac{\pi \nu}{2} .
$$

Because $I_{\nu}(x)$ is a continuous function of $\nu$ in a neighborhood of $\nu=1$, we can let $\nu \rightarrow 1$ in (3.12), and, with the use of L'Hôspital's rule, deduce the following lemma.

LEMma 3.5. We have

$$
\int_{0}^{\infty} I_{1}(x) d x=0 .
$$

We require two well-known Fourier series, which can be found in the tables of Gradshteyn and Ryzhik [17, p. 45, formula 1.441 , no. 1; p. 46, formula 1.441, no. 2].

Lemma 3.6. For any real number $x$,

$$
-\sum_{m=1}^{\infty} \frac{\sin (2 \pi m x)}{\pi m}= \begin{cases}0, & \text { if } x \text { is an integer, } \\ x-[x]-\frac{1}{2}, & \text { if } x \text { is not an integer. }\end{cases}
$$

LEMma 3.7. For $0<x<1$,

$$
\sum_{j=1}^{\infty} \frac{\cos (2 \pi j x)}{j}=-\log (2 \sin (\pi x)) .
$$


As indicated in Section 2, numerical calculations appear to indicate that perhaps an unknown function, say, $G(\theta)$, needs to be inserted into (2.10) in order to obtain a valid identity.

We are now ready to state a possibly corrected version of Ramanujan's second formula, Entry 2.2 , which we, at this point, optimistically label a theorem.

THEOREM 3.8. Let $F(x)$ be defined by (2.2), and let $I_{1}(z)$ be defined by (2.9). Suppose that an appropriate function $G(\theta)$ exists such that

$$
\int_{0}^{1 / 2} G(\theta) \cos (2 \pi n \theta) d \theta=-\frac{1}{8}
$$

Then, for $x>0$ and $0<\theta<1$,

$$
\begin{aligned}
\sum_{n=1}^{\infty} F\left(\frac{x}{n}\right) \cos (2 \pi n \theta) & =\frac{1}{4}-x \log (2 \sin (\pi \theta))+G(\theta) \\
+ & \frac{1}{2} \sqrt{x} \sum_{m=1}^{\infty} \sum_{n=0}^{\infty}\left\{\frac{I_{1}(4 \pi \sqrt{m(n+\theta) x})}{\sqrt{m(n+\theta)}}+\frac{I_{1}(4 \pi \sqrt{m(n+1-\theta) x})}{\sqrt{m(n+1-\theta)}}\right\} .
\end{aligned}
$$

Proof. (incomplete) We calculate the Fourier cosine series of

$$
f(\theta):=G(\theta)+\frac{1}{2} \sqrt{x} \sum_{m=1}^{\infty} \sum_{n=0}^{\infty}\left\{\frac{I_{1}(4 \pi \sqrt{m(n+\theta) x})}{\sqrt{m(n+\theta)}}+\frac{I_{1}(4 \pi \sqrt{m(n+1-\theta) x})}{\sqrt{m(n+1-\theta)}}\right\} .
$$

Write

$$
f(\theta)=\frac{1}{2} a_{0}+\sum_{j=1}^{\infty} a_{j} \cos (2 \pi j \theta)
$$

where, for $j \geq 0$,

$$
a_{j}=4 \int_{0}^{1 / 2} f(\theta) \cos (2 \pi j \theta) d \theta
$$

We first calculate $a_{0}$. To that end, by (3.14) and the assumption that changing the order of summation and integration below is justified,

$$
\begin{aligned}
a_{0}= & 4 \int_{0}^{1 / 2} f(\theta) d \theta=4 \int_{0}^{1 / 2} G(\theta) d \theta \\
& +2 \sqrt{x} \int_{0}^{1 / 2} \sum_{m=1}^{\infty} \sum_{n=0}^{\infty}\left\{\frac{I_{1}(4 \pi \sqrt{m(n+\theta) x})}{\left.\sqrt{m(n+\theta)}+\frac{I_{1}(4 \pi \sqrt{m(n+1-\theta) x})}{\sqrt{m(n+1-\theta)}}\right\} d \theta}\right. \\
= & -\frac{1}{2}+2 \sqrt{x} \sum_{m=1}^{\infty} \sum_{n=0}^{\infty} \int_{0}^{1 / 2}\left\{\frac{I_{1}(4 \pi \sqrt{m(n+\theta) x})}{\sqrt{m(n+\theta)}}+\frac{I_{1}(4 \pi \sqrt{m(n+1-\theta) x})}{\sqrt{m(n+1-\theta)}}\right\} d \theta .
\end{aligned}
$$

On the far right side above, write the integral of the sum of the two functions in the integrands as the sum of the integrals of the functions taken individually. In the first integral, make the change of variable $u=4 \pi \sqrt{m(n+\theta) x}$, and in the second, set $u=4 \pi \sqrt{m(n+1-\theta) x}$. Hence,

$$
\begin{aligned}
a_{0} & =-\frac{1}{2}+\frac{1}{\pi} \sum_{m=1}^{\infty} \sum_{n=0}^{\infty} \frac{1}{m}\left\{\int_{4 \pi \sqrt{m n x}}^{4 \pi \sqrt{m(n+1 / 2) x}} I_{1}(u) d u-\int_{4 \pi \sqrt{m(n+1) x}}^{4 \pi \sqrt{m(n+1 / 2) x}} I_{1}(u) d u\right\} \\
& =-\frac{1}{2}+\frac{1}{\pi} \sum_{m=1}^{\infty} \sum_{n=0}^{\infty} \frac{1}{m} \int_{4 \pi \sqrt{m n x}}^{4 \pi \sqrt{m(n+1) x}} I_{1}(u) d u \\
& =-\frac{1}{2}+\frac{1}{\pi} \sum_{m=1}^{\infty} \frac{1}{m} \int_{0}^{\infty} I_{1}(u) d u=-\frac{1}{2},
\end{aligned}
$$

upon the use of Lemma 3.5. 
Next, for $j \geq 1$, by (3.14), assuming that we can invert the order of integration and summation below, we find that

$$
\begin{aligned}
a_{j}= & 4 \int_{0}^{1 / 2} G(\theta) d \theta+2 \sqrt{x} \int_{0}^{1 / 2} \sum_{m=1}^{\infty} \sum_{n=0}^{\infty}\left\{\frac{I_{1}(4 \pi \sqrt{m(n+\theta) x})}{\sqrt{m(n+\theta)}}\right. \\
& \left.+\frac{I_{1}(4 \pi \sqrt{m(n+1-\theta) x})}{\sqrt{m(n+1-\theta)}}\right\} \cos (2 \pi j \theta) d \theta \\
= & -\frac{1}{2}+2 \sqrt{x} \sum_{m=1}^{\infty} \sum_{n=0}^{\infty} \int_{0}^{1 / 2}\left\{\frac{I_{1}(4 \pi \sqrt{m(n+\theta) x})}{\sqrt{m(n+\theta)}}\right. \\
& \left.+\frac{I_{1}(4 \pi \sqrt{m(n+1-\theta) x})}{\sqrt{m(n+1-\theta)}}\right\} \cos (2 \pi j \theta) d \theta .
\end{aligned}
$$

As above, we write the integral on the far right side above as a sum of two integrals and make the same changes of variables as we did in the calculation of $a_{0}$. For brevity below, set

$$
\alpha_{m}:=\frac{j}{8 \pi m x} .
$$

Accordingly, we find that

$$
\begin{aligned}
a_{j}= & -\frac{1}{2}+\frac{1}{\pi} \sum_{m=1}^{\infty} \sum_{n=0}^{\infty} \frac{1}{m}\left\{\int_{4 \pi \sqrt{m n x}}^{4 \pi \sqrt{m(n+1 / 2) x}} I_{1}(u) \cos \left(2 \pi j\left(\frac{u^{2}}{16 \pi^{2} m x}-n\right)\right) d u\right. \\
& \left.-\int_{4 \pi \sqrt{m(n+1) x}}^{4 \pi \sqrt{m(n+1 / 2) x}} I_{1}(u) \cos \left(2 \pi j\left(n+1-\frac{u^{2}}{16 \pi^{2} m x}\right)\right) d u\right\} \\
= & -\frac{1}{2}+\frac{1}{\pi} \sum_{m=1}^{\infty} \sum_{n=0}^{\infty} \frac{1}{m}\left\{\int_{4 \pi \sqrt{m n x}}^{4 \pi \sqrt{m(n+1 / 2) x}} I_{1}(u) \cos \left(\alpha_{m} u^{2}\right) d u\right. \\
& \left.-\int_{4 \pi \sqrt{m(n+1) x}}^{4 \pi \sqrt{m(n+1 / 2) x}} I_{1}(u) \cos \left(\alpha_{m} u^{2}\right) d u\right\} \\
= & -\frac{1}{2}+\frac{1}{\pi} \sum_{m=1}^{\infty} \sum_{n=0}^{\infty} \frac{1}{m} \int_{4 \pi \sqrt{m n x}}^{4 \pi \sqrt{m(n+1) x}} I_{1}(u) \cos \left(\alpha_{m} u^{2}\right) d u \\
= & -\frac{1}{2}+\frac{1}{\pi} \sum_{m=1}^{\infty} \frac{1}{m} \int_{0}^{\infty} I_{1}(u) \cos \left(\alpha_{m} u^{2}\right) d u .
\end{aligned}
$$

We now apply Lemma 3.4 above with $c=1$ and $b=\alpha_{m}$, as given in (3.19). Recall that $A$ is given by (3.3), and so $\alpha_{m} A=\frac{1}{8}$. Hence, also using Lemma 3.6, we find that

$$
\begin{aligned}
a_{j} & =-\frac{1}{2}+\frac{1}{\pi} \sum_{m=1}^{\infty} \frac{1}{m} \sin (2 \pi m x / j) \\
& =-\frac{1}{2}+ \begin{cases}0, & \text { if } \frac{x}{j} \text { is an integer, } \\
-\left(\frac{x}{j}-\left[\frac{x}{j}\right]-\frac{1}{2}\right), & \text { if } \frac{x}{j} \text { is not an integer, }\end{cases} \\
& = \begin{cases}{\left[\frac{x}{j}\right]-\frac{1}{2}-\frac{x}{j},} & \text { if } \frac{x}{j} \text { is an integer, } \\
{\left[\frac{x}{j}\right]-\frac{x}{j},} & \text { if } \frac{x}{j} \text { is not an integer, }\end{cases}
\end{aligned}
$$




$$
=F\left(\frac{x}{j}\right)-\frac{x}{j}
$$

At last, putting (3.18) and (3.21) into (3.17) and employing Lemma 3.7, we deduce that

$$
\begin{aligned}
f(\theta) & =-\frac{1}{4}+\sum_{j=1}^{\infty} F\left(\frac{x}{j}\right) \cos (2 \pi j \theta)-x \sum_{j=1}^{\infty} \frac{\cos (2 \pi j \theta)}{j} \\
& =-\frac{1}{4}+\sum_{j=1}^{\infty} F\left(\frac{x}{j}\right) \cos (2 \pi j \theta)+x \log (2 \sin (\pi \theta)) .
\end{aligned}
$$

A simple rearrangement of (3.22) shows that it is equivalent to (2.10). The justification of the inversion of double summation and integration at the beginning of the proof is a consequence of our calculations in (3.20) and (3.21), which yield a boundedly convergent series. Thus, the proof is complete.

\section{A Connection of (2.10) with Voronoï's Formula (2.13)}

As indicated in Section 2, we now make firm the connection between the two formulas heading this section in the following theorem.

Theorem 4.1. Recall that $I_{1}(z)$ is defined by (2.9). Then, for $x>0$,

$$
\lim _{\theta \rightarrow 0^{+}}\left(\frac{1}{2} \sum_{m=0}^{\infty}\left(\frac{x}{m \theta}\right)^{1 / 2} I_{1}(4 \pi \sqrt{m x \theta})-x \log (2 \sin (\pi \theta))\right)=x(\log x+2 \gamma-1),
$$

where $\gamma$ denotes Euler's constant.

Proof. First observe that, by writing $n=m k$, we can rewrite (2.13) in the form

$$
\sum_{n \leq x}^{\prime} d(n)=x(\log x+(2 \gamma-1))+\frac{1}{4}+\sum_{m=1}^{\infty} \sum_{k=1}^{\infty}\left(\frac{x}{m k}\right)^{1 / 2} I_{1}(4 \pi \sqrt{m k x}) .
$$

From the right-hand side of (2.10),

$$
\begin{aligned}
& \lim _{\theta \rightarrow 0^{+}}\left(-x \log (2 \sin (\pi \theta))+\frac{1}{2} \sqrt{x} \sum_{m=1}^{\infty} \sum_{n=0}^{\infty}\left\{\frac{I_{1}(4 \pi \sqrt{m(n+\theta) x})}{\sqrt{m(n+\theta)}}+\frac{I_{1}(4 \pi \sqrt{m(n+1-\theta) x})}{\sqrt{m(n+1-\theta)}}\right\}\right) \\
= & \lim _{\theta \rightarrow 0^{+}}\left(-x \log (2 \sin (\pi \theta))+\frac{1}{2} \sqrt{x} \sum_{m=1}^{\infty} \frac{I_{1}(4 \pi \sqrt{m \theta x})}{\sqrt{m \theta}}+\frac{1}{2} \sqrt{x} \sum_{m=1}^{\infty} \frac{I_{1}(4 \pi \sqrt{m \theta x})}{\sqrt{m(1-\theta)}}\right. \\
& +\frac{1}{2} \sqrt{x} \sum_{m=1}^{\infty} \sum_{n=1}^{\infty}\left\{\frac{I_{1}(4 \pi \sqrt{m(n+\theta) x})}{\left.\left.\sqrt{m(n+\theta)}+\frac{I_{1}(4 \pi \sqrt{m(n+1-\theta) x})}{\sqrt{m(n+1-\theta)}}\right\}\right)}\right. \\
= & \lim _{\theta \rightarrow 0^{+}}\left(-x \log (2 \sin (\pi \theta))+\frac{1}{2} \sqrt{x} \sum_{m=1}^{\infty} \frac{I_{1}(4 \pi \sqrt{m \theta x})}{\sqrt{m \theta}}\right)+\sqrt{x} \sum_{m=1}^{\infty} \sum_{n=1}^{\infty} \frac{I_{1}(4 \pi \sqrt{m n x})}{\sqrt{m n}} \\
= & \lim _{\theta \rightarrow 0^{+}}\left(-x \log (2 \sin (\pi \theta))+\frac{1}{2} \sqrt{x} \sum_{m=1}^{\infty} \frac{I_{1}(4 \pi \sqrt{m \theta x})}{\sqrt{m \theta}}\right)
\end{aligned}
$$

$$
+\sum_{n \leq x}^{\prime} d(n)-x(\log x+(2 \gamma-1))-\frac{1}{4}
$$


by (4.2). On the other hand, from the left-hand side of (2.10), upon the use of an elementary formula for $\sum_{n \leq x}{ }^{\prime} d(n)[\mathbf{2 5}$, p. 101],

$$
\begin{aligned}
\lim _{\theta \rightarrow 0^{+}} \sum_{n=1}^{\infty} F\left(\frac{x}{n}\right) \cos (2 \pi n \theta) & =\sum_{n \leq x} F\left(\frac{x}{n}\right) \\
& = \begin{cases}\sum_{n \leq x}\left[\frac{x}{n}\right]-\frac{1}{2} d(x), & \text { if } x \text { is an integer, } \\
\sum_{n \leq x}\left[\frac{x}{n}\right], & \text { if } x \text { is not an integer, }\end{cases} \\
& =\sum_{n \leq x}^{\prime} d(n) .
\end{aligned}
$$

Combining (4.3) with (4.4), substituting the results in (2.10), and simplifying, we deduce (4.1) to complete the proof.

\section{Evidence for Divergence}

We begin our investigation into the behavior of (2.10) by carrying out numerical computations to check for both convergence of the double series as well as for agreement of the left- and righthand sides. Define

$$
L(x, \theta):=-\frac{1}{4}+x \log (2 \sin (\pi \theta))+\sum_{n=1}^{\infty} F\left(\frac{x}{n}\right) \cos (2 n \pi \theta)
$$

and

$$
R(x, \theta, M, N):=\frac{1}{2} \sqrt{x} \sum_{m=1}^{M} \sum_{n=0}^{N}\left(\frac{I_{1}(4 \pi \sqrt{m(n+\theta) x})}{\sqrt{m(n+\theta)}}+\frac{I_{1}(4 \pi \sqrt{m(n+1-\theta) x})}{\sqrt{m(n+1-\theta)}}\right) .
$$

The computations were done on Pari-GP version 2.3.1, computed with a real-precision of 19 digits and checked at a precision of 28 digits. Our results are summarized in Tables 1 and 2. Table 1 shows that although the values of $R(x, \theta, M, N)$ are somewhat close to the value of $L(x, \theta)$ (to one significant digit), they do not seem to stabilize as we increase the upper limits on the partial sums, even after summing tens of thousands of terms. These results cast doubt on whether the right-hand side of (2.10) is indeed correct, or even converges.

Our next plan of attack, given the evidence from the Fourier analysis, is to try to determine the behavior of the missing function of $\theta$, if it exists. To that end, we computed the partial sums at several values of $\theta, M$, and $N$, and noted the differences between the two sides of (2.10) (see Table 2). The data suggest that the difference $L-R$ is not necessarily stable as we increase the number of summands, and that if such a function of $\theta$ exists, it has a singularity at $\theta=0$. It may be possible to obtain more insightful results if we carried the calculations further, but evaluations of sums of Bessel functions are computationally expensive, especially for two-dimensional sums.

We now examine the convergence properties of (2.10). The asymptotic formulas (2.11) and (2.12) allow us to reduce our analysis to a double sum involving the sine function, given by the following easily proved theorem.

TheOREM 5.1. For $0<\theta<1$ and $x>0$, the sum

$$
\sum_{m=1}^{\infty} \sum_{n=0}^{\infty}\left(\frac{I_{1}(4 \pi \sqrt{m(n+\theta) x})}{\sqrt{m(n+\theta)}}+\frac{I_{1}(4 \pi \sqrt{m(n+1-\theta) x})}{\sqrt{m(n+1-\theta)}}\right)
$$

converges if and only if the sum

$$
\sum_{m=1}^{\infty} \sum_{n=0}^{\infty}\left(\frac{\sin \left(4 \pi \sqrt{m(n+\theta) x}-\frac{3}{4} \pi\right)}{m^{3 / 4}(n+\theta)^{3 / 4}}+\frac{\sin \left(4 \pi \sqrt{m(n+1-\theta) x}-\frac{3}{4}\right) \pi}{m^{3 / 4}(n+1-\theta)^{3 / 4}}\right)
$$

converges. 
TABLE 1

\begin{tabular}{|c|c|c|c|c|c|}
\hline$x$ & $\theta$ & $M$ & $N$ & $L(x, \theta)$ & $R(x, \theta, M, N)$ \\
\hline \multirow{3}{*}{0.5} & \multirow{3}{*}{0.5} & \multirow{3}{*}{20} & 200 & \multirow{3}{*}{0.09657} & 0.10563 \\
\hline & & & 500 & & 0.10237 \\
\hline & & & 1000 & & 0.11033 \\
\hline \multirow{3}{*}{0.5} & \multirow{3}{*}{0.5} & \multirow{3}{*}{50} & 200 & \multirow{3}{*}{0.09657} & 0.09394 \\
\hline & & & 500 & & 0.09140 \\
\hline & & & 1000 & & 0.09834 \\
\hline \multirow{3}{*}{0.5} & \multirow{3}{*}{0.5} & 200 & \multirow{3}{*}{20} & \multirow{3}{*}{0.09657} & 0.13265 \\
\hline & & 500 & & & 0.14249 \\
\hline & & 1000 & & & 0.11553 \\
\hline \multirow{3}{*}{0.5} & \multirow{3}{*}{0.5} & 200 & \multirow{3}{*}{50} & \multirow{3}{*}{0.09657} & 0.11492 \\
\hline & & 500 & & & 0.12499 \\
\hline & & 1000 & & & 0.09840 \\
\hline \multirow{3}{*}{1.75} & \multirow{3}{*}{0.25} & \multirow{3}{*}{20} & 200 & \multirow{3}{*}{0.35650} & 0.35218 \\
\hline & & & 500 & & 0.35655 \\
\hline & & & 1000 & & 0.35055 \\
\hline \multirow{3}{*}{1.75} & \multirow{3}{*}{0.25} & \multirow{3}{*}{50} & 200 & \multirow{3}{*}{0.35650} & 0.39195 \\
\hline & & & 500 & & 0.39685 \\
\hline & & & 1000 & & 0.39056 \\
\hline \multirow{3}{*}{1.75} & \multirow{3}{*}{0.25} & 200 & \multirow{3}{*}{20} & \multirow{3}{*}{0.35650} & 0.34695 \\
\hline & & 500 & & & 0.36495 \\
\hline & & 1000 & & & 0.34645 \\
\hline \multirow{3}{*}{1.75} & \multirow{3}{*}{0.25} & 200 & \multirow{3}{*}{50} & \multirow{3}{*}{0.35650} & 0.32983 \\
\hline & & 500 & & & 0.34832 \\
\hline & & 1000 & & & 0.32934 \\
\hline
\end{tabular}

Suppose that minus signs separated the Bessel functions on the right-hand side of (2.10). The same analysis shows that

$$
\sum_{m=1}^{\infty} \sum_{n=0}^{\infty}\left(\frac{I_{1}(4 \pi \sqrt{m(n+\theta) x})}{\sqrt{m(n+\theta)}}-\frac{I_{1}(4 \pi \sqrt{m(n+1-\theta) x})}{\sqrt{m(n+1-\theta)}}\right)
$$

converges if and only if the sum

$$
\sum_{m=1}^{\infty} \sum_{n=0}^{\infty}\left(\frac{\sin \left(4 \pi \sqrt{m(n+\theta) x}-\frac{3}{4} \pi\right)}{m^{3 / 4}(n+\theta)^{3 / 4}}-\frac{\sin \left(4 \pi \sqrt{m(n+1-\theta) x}-\frac{3}{4} \pi\right)}{m^{3 / 4}(n+1-\theta)^{3 / 4}}\right)
$$

converges. Analogous theorems are easily proved for the corresponding sums with the reverse order of summation, as well as for the right-hand side of (2.3), with sine replaced by cosine. Since trigonometric functions can be evaluated much more efficiently than Bessel functions, we can compute the sums (5.2) and (5.4) to many more terms than we could for $R(x, \theta, M, N)$. Define

$$
S_{ \pm}(x, \theta, M, N):=\sum_{m=1}^{M} \sum_{n=0}^{N}\left(\frac{\sin \left(4 \pi \sqrt{m(n+\theta) x}-\frac{3}{4} \pi\right)}{m^{3 / 4}(n+\theta)^{3 / 4}} \pm \frac{\sin \left(4 \pi \sqrt{m(n+\theta) x}-\frac{3}{4} \pi\right)}{m^{3 / 4}(n+\theta)^{3 / 4}}\right),
$$

and

$$
C_{-}(x, \theta, M, N):=\sum_{m=1}^{M} \sum_{n=0}^{N}\left(\frac{\cos \left(4 \pi \sqrt{m(n+\theta) x}-\frac{3}{4} \pi\right)}{m^{3 / 4}(n+\theta)^{3 / 4}}-\frac{\cos \left(4 \pi \sqrt{m(n+\theta) x}-\frac{3}{4} \pi\right)}{m^{3 / 4}(n+\theta)^{3 / 4}}\right) .
$$

When $\theta=\frac{1}{2}$, both $S_{-}$and $C_{-}$trivially converge as $M, N \rightarrow \infty$ because all the terms are identically equal to 0 . Therefore, in order to compare the behavior with that of $S_{+}$, we carried out computations for $\theta=\frac{1}{4}$ (Table 3). Recall that Berndt and Zaharescu [14] proved that $C_{-}$ converges if we let $M \rightarrow \infty$ first. Table 3 shows that in all three cases, for fixed $M$, increasing $N$ does not significantly change the value of the sum. On the other hand, when $N$ is fixed and 
TABLE 2

\begin{tabular}{|c|c|c|c|c|c|c|c|}
\hline$x$ & $\theta$ & $M$ & $N$ & $R(x, \theta, M, N)-L(x, \theta)$ & $M$ & $N$ & $R(x, \theta, M, N)-L(x, \theta)$ \\
\hline \multirow{3}{*}{0.5} & \multirow{3}{*}{0.05} & \multirow{3}{*}{20} & 200 & 0.40135 & 200 & \multirow{3}{*}{20} & 0.21536 \\
\hline & & & 500 & 0.39818 & 500 & & -0.03815 \\
\hline & & & 800 & 0.40067 & 800 & & -0.14057 \\
\hline \multirow{3}{*}{0.5} & \multirow{3}{*}{0.10} & \multirow{3}{*}{20} & 200 & -0.12100 & 200 & \multirow{3}{*}{20} & 0.11169 \\
\hline & & & 500 & -0.12419 & 500 & & -0.03952 \\
\hline & & & 800 & -0.12171 & 800 & & 0.00101 \\
\hline \multirow{3}{*}{0.5} & \multirow{3}{*}{0.15} & \multirow{3}{*}{20} & 200 & 0.06303 & 200 & \multirow{3}{*}{20} & -0.00907 \\
\hline & & & 500 & 0.05983 & 500 & & 0.05608 \\
\hline & & & 800 & 0.06231 & 800 & & 0.04562 \\
\hline \multirow{3}{*}{0.5} & \multirow{3}{*}{0.20} & \multirow{3}{*}{20} & 200 & -0.08595 & 200 & \multirow{3}{*}{20} & -0.03302 \\
\hline & & & 500 & -0.08917 & 500 & & 0.03098 \\
\hline & & & 800 & -0.08668 & 800 & & -0.01444 \\
\hline \multirow{3}{*}{0.5} & \multirow{3}{*}{0.25} & \multirow{3}{*}{20} & 200 & 0.04009 & 200 & \multirow{3}{*}{20} & 0.01474 \\
\hline & & & 500 & 0.03686 & 500 & & 0.01102 \\
\hline & & & 800 & 0.03935 & 800 & & 0.00430 \\
\hline \multirow{3}{*}{0.5} & \multirow{3}{*}{0.30} & \multirow{3}{*}{20} & 200 & 0.05794 & 200 & \multirow{3}{*}{20} & -0.00284 \\
\hline & & & 500 & 0.05470 & 500 & & 0.05013 \\
\hline & & & 800 & 0.05719 & 800 & & 0.01133 \\
\hline \multirow{3}{*}{0.5} & \multirow{3}{*}{0.35} & \multirow{3}{*}{20} & 200 & -0.02133 & 200 & \multirow{3}{*}{20} & 0.01033 \\
\hline & & & 500 & -0.02458 & 500 & & 0.02078 \\
\hline & & & 800 & -0.02209 & 800 & & 0.03522 \\
\hline \multirow{3}{*}{0.5} & \multirow{3}{*}{0.40} & & 200 & -0.03525 & 200 & & 0.02988 \\
\hline & & 20 & 500 & -0.03851 & 500 & 20 & 0.02096 \\
\hline & & & 800 & -0.03601 & 800 & & 0.03072 \\
\hline & & & 200 & -0.00540 & 200 & & 0.02512 \\
\hline 0.5 & 0.45 & 20 & 500 & -0.00866 & 500 & 20 & 0.04543 \\
\hline & & & 800 & -0.00617 & 800 & & 0.01503 \\
\hline & & & 200 & 0.00906 & 200 & & 0.03608 \\
\hline 0.5 & 0.50 & 20 & 500 & 0.00580 & 500 & 20 & 0.04592 \\
\hline & & & 800 & 0.00829 & 800 & & 0.03912 \\
\hline
\end{tabular}

$M$ increases, we see that the value of the sum fluctuates, although $S_{-}$appears to be more stable than $S_{+}$. In fact, aside from the strange evaluation at $M=1000, S_{-}$seems to be convergent as $M$ and $N$ become large.

However, these calculations do not necessarily indicate that $S_{+}$is not convergent. Indeed, we know that $C_{-}$is convergent, and the fluctuations of the values of $S_{+}$do not seem much different from those of $C_{-}$. The apparent behavior of these sums as we increase $M$ and $N$ do provide hints for attacking the convergence problem. We see that the main contributions for the sums come from the index $m$, and increasing the value of $N$ for any fixed $M$ has little effect on the value of the sums. Therefore, we expect that the sum indexed by $n$ would be small and easier to handle. Thus, it may be instructive to attempt to prove convergence of (2.10) with the order of summation reversed, as was done for (2.3) in [14].

\section{First Attempts to Find Meanings for Entries 2.4 and 2.3}

Our first attempts to prove Entries 2.4 and 2.3 depend upon the Voronoï summation formula. We only briefly sketch the background and hypotheses needed for the statement of the Voronoï summation formula. For complete details, see the papers $[\mathbf{4}],[\mathbf{5}],[\mathbf{6}]$, and [15]. 
TABLE 3

\begin{tabular}{|c|c|c|c|c|c|c|}
\hline$x$ & $\theta$ & $M$ & $N$ & $S_{+}(x, \theta, M, N)$ & $S_{-}(x, \theta, M, N)$ & $C_{-}(x, \theta, M, N)$ \\
\hline \multirow{5}{*}{0.5} & \multirow{5}{*}{0.25} & \multirow{5}{*}{20} & 200 & 0.45625 & 1.76337 & -2.09380 \\
\hline & & & 500 & 0.48983 & 1.74244 & -2.09078 \\
\hline & & & 1000 & 0.40621 & 1.75015 & -2.09843 \\
\hline & & & 5000 & 0.43785 & 1.75325 & -2.09527 \\
\hline & & & 10000 & 0.40369 & 1.75295 & -2.09536 \\
\hline \multirow{5}{*}{0.5} & \multirow{5}{*}{0.25} & \multirow{5}{*}{50} & 200 & 1.21740 & 2.23086 & -1.91890 \\
\hline & & & 500 & 1.24370 & 2.20498 & -1.91799 \\
\hline & & & 1000 & 1.17060 & 2.21346 & -1.92325 \\
\hline & & & 5000 & 1.19867 & 2.21571 & -1.92084 \\
\hline & & & 10000 & 1.16151 & 2.21542 & -1.92112 \\
\hline \multirow{5}{*}{0.5} & \multirow{5}{*}{0.25} & \multirow{5}{*}{200} & 200 & 0.99062 & 2.30836 & -1.63579 \\
\hline & & & 500 & 1.02271 & 2.28440 & -1.62905 \\
\hline & & & 1000 & 0.95210 & 2.29101 & -1.63351 \\
\hline & & & 5000 & 0.97957 & 2.29339 & -1.63141 \\
\hline & & & 10000 & 0.94061 & 2.29306 & -1.63199 \\
\hline \multirow{5}{*}{0.5} & \multirow{5}{*}{0.25} & \multirow{5}{*}{500} & 200 & 1.03468 & 2.26829 & -1.27184 \\
\hline & & & 500 & 1.06464 & 2.24093 & -1.26746 \\
\hline & & & 1000 & 0.99557 & 2.24744 & -1.27037 \\
\hline & & & 5000 & 1.02308 & 2.24982 & -1.26781 \\
\hline & & & 10000 & 0.98523 & 2.24957 & -1.26813 \\
\hline \multirow{5}{*}{0.5} & \multirow{5}{*}{0.25} & \multirow{5}{*}{1000} & 200 & 0.70896 & 1.78790 & -1.40521 \\
\hline & & & 500 & 0.73881 & 1.76293 & -1.40061 \\
\hline & & & 1000 & 0.66918 & 1.76867 & -1.40406 \\
\hline & & & 5000 & 0.69728 & 1.77120 & -1.40092 \\
\hline & & & 10000 & 0.65960 & 1.77070 & -1.40120 \\
\hline \multirow{5}{*}{0.5} & \multirow{5}{*}{0.25} & 200 & & 0.99062 & 2.30836 & -1.63579 \\
\hline & & 500 & & 1.03468 & 2.26829 & -1.27184 \\
\hline & & 1000 & 200 & 0.70896 & 1.78790 & -1.40521 \\
\hline & & 5000 & & 1.01898 & 2.13973 & -1.49686 \\
\hline & & 10000 & & 0.94203 & 2.11789 & -1.26361 \\
\hline \multirow{5}{*}{0.5} & \multirow{5}{*}{0.25} & 200 & & 1.02271 & 2.28440 & -1.62905 \\
\hline & & 500 & & 1.06464 & 2.24093 & -1.26746 \\
\hline & & 1000 & 500 & 0.73881 & 1.76293 & -1.40061 \\
\hline & & 5000 & & 1.04886 & 2.11597 & -1.49290 \\
\hline & & 10000 & & 0.97231 & 2.09375 & -1.25932 \\
\hline \multirow{5}{*}{0.5} & \multirow{5}{*}{0.25} & 200 & & 0.95210 & 2.29101 & -1.63351 \\
\hline & & 500 & & 0.99557 & 2.24744 & -1.27037 \\
\hline & & 1000 & 1000 & 0.66918 & 1.76867 & -1.40406 \\
\hline & & 5000 & & 0.97889 & 2.12250 & -1.49634 \\
\hline & & 10000 & & 0.90268 & 2.09993 & -1.26273 \\
\hline
\end{tabular}

Let $s=\sigma+i t$, with $\sigma$ and $t$ real, and let

$$
\phi(s):=\sum_{n=1}^{\infty} a(n) \lambda_{n}^{-s} \quad \text { and } \quad \psi(s):=\sum_{n=1}^{\infty} b(n) \mu_{n}^{-s}, \quad 0<\lambda_{n}, \mu_{n} \rightarrow \infty
$$

be two Dirichlet series with abscissas of absolute convergence $\sigma_{a}$ and $\sigma_{a}^{*}$, respectively. Let $r>0$, and suppose that $\phi(s)$ and $\psi(s)$ satisfy a functional equation of the type

$$
\Gamma(s) \phi(s)=\Gamma(r-s) \psi(r-s) .
$$


For such arithmetical functions, K. Chandrasekharan and R. Narasimhan [15] and Berndt [4], [5] have established theorems providing representations for the Riesz sum

$$
A_{q}(x):=\frac{1}{\Gamma(q+1)} \sum_{\lambda_{n} \leq x}^{\prime} a(n)\left(x-\lambda_{n}\right)^{q},
$$

where $q>2 \sigma_{a}^{*}-r-\frac{1}{2}, x>0$, and the prime $/$ indicates that if $q=0$ and $\lambda_{n}=x$ for some positive integer $n, a(n)$ is to be multiplied by $\frac{1}{2}$. Define also

$$
Q_{q}(x):=\int_{C_{q}} \frac{\Gamma(s) \phi(s) x^{s+q}}{\Gamma(s+q+1)} d s
$$

where $C_{q}$ is a simple closed curve(s) containing the integrand's poles on its interior. Set $Q(x):=$ $Q_{0}(x)$.

The Voronoï summation formula in its original form with $a(n)=d(n)$, where $d(n)$ denotes the number of positive divisors of the positive integer $n$, was first proved by M. G. Voronoi in 1904 [30]. Since then "Voronoï summation formulas" have been established for a variety of arithmetical functions under various hypotheses. In particular, Berndt [6] established various versions of the Voronoï summation formula, including the following theorem from [6, p. 142, Thm. 1], where several references to the literature on Voronoï summation formulas can be found.

Theorem 6.1. Let $f \in C^{(1)}(0, \infty)$. Then, if $0<a<\lambda_{1}<x<\infty$,

$$
\sum_{\lambda_{n} \leq x}^{\prime} a(n) f\left(\lambda_{n}\right)=\int_{a}^{x} Q^{\prime}(t) f(t) d t+\sum_{n=1}^{\infty} b(n) \int_{a}^{x}\left(\frac{t}{\mu_{n}}\right)^{(r-1) / 2} J_{r-1}\left(2 \sqrt{\mu_{n} t}\right) f(t) d t,
$$

where, as above, the prime 1 on the summation sign on the left-hand side indicates that if $x=\lambda_{n}$, for some integer $n$, then only $\frac{1}{2} a(n) f\left(\lambda_{n}\right)$ is counted, and where $J_{\nu}(x)$ denotes the ordinary Bessel function of order $\nu$.

This is the simplest theorem of this sort. The two applications that we make of Theorem 6.1 are formal in the sense that there are no versions of the Voronoï summation formula that would ensure the validity of our applications; indeed, as we remarked above, both (2.16) and (2.17) contain divergent series. We think that Ramanujan likely discovered some version of the Voronoï summation formula for $a(n)=\sigma_{k}(n)$, but he apparently had neither established a precise version nor conditions for its validity. We next see how Ramanujan might have been led to the two entries above.

In order to avoid possible confusion, we are going to replace $s$ by $k$ in our attempts to prove (2.16) and (2.17). It is well-known and easy to prove that, for any real number $k$,

$$
\zeta(s) \zeta(s-k)=\sum_{n=1}^{\infty} \frac{\sigma_{k}(n)}{n^{s}}, \quad \sigma>\sup \{1, k+1\} .
$$

Then with the use of the functional equation $(2.20)$ for $\zeta(s)$, it is not difficult to show that $[\mathbf{1 5}$, p. 17], if $k$ is an odd integer,

$$
(2 \pi)^{-s} \Gamma(s) \zeta(s) \zeta(k-s)=(-1)^{(k+1) / 2}(2 \pi)^{-(k+1-s)} \Gamma(k+1-s) \zeta(k+1-s) \zeta(1-s) .
$$

Thus, in the settings (6.1) and (6.5), we have

$$
\begin{gathered}
a(n)=\sigma_{k}(n), \quad b(n)=(-1)^{(k+1) / 2} \sigma_{k}(n), \quad k \text { odd }, \\
\lambda_{n}=\mu_{n}=2 \pi n, \quad n \geq 1, \quad r=k+1 .
\end{gathered}
$$

Furthermore, $Q(x)$ is the sum of the residues of

$$
\frac{(2 \pi)^{-s} \zeta(s) \zeta(s-k) x^{s}}{s}
$$

taken over all its poles, which are at $s=1, s=k+1$, and $s=0$. Since $\zeta(s)$ has a simple pole at $s=1$ with residue 1 and $[\mathbf{2 9}$, p. 19]

$$
\zeta(0)=-\frac{1}{2},
$$


we find that

$$
Q(x)=-\frac{1}{2} \zeta(-k)+\frac{\zeta(1-k) x}{2 \pi}+\frac{\zeta(k+1) x^{k+1}}{(2 \pi)^{k+1}(k+1)} .
$$

It follows that

$$
Q^{\prime}(x)=\frac{\zeta(1-k)}{2 \pi}+\frac{\zeta(k+1) x^{k}}{(2 \pi)^{k+1}} .
$$

We first examine (2.17). In our formal application of (6.3), we clearly should set $a=0, x=\infty$, and $f(t)=\sin (\alpha t /(2 \pi))$. In order to apply (6.3), we need to employ the integral evaluation [17, p. 773 , formula 6.728, no. 5$]$

$$
\int_{0}^{\infty} x^{k+1} J_{k}(b x) \sin \left(a x^{2}\right) d x=\frac{b^{k}}{(2 a)^{k+1}} \cos \left(\frac{b^{2}}{4 a}-\frac{k \pi}{2}\right) .
$$

Hence, using (6.10), we find that

$$
\begin{aligned}
\int_{0}^{\infty} t^{k / 2} J_{k}(2 \sqrt{2 \pi n t}) \sin \left(\frac{\alpha t}{2 \pi}\right) d t & =2 \int_{0}^{\infty} u^{k+1} J_{k}\left(2 \sqrt{\mu_{n}} u\right) \sin \left(\frac{\alpha u^{2}}{2 \pi}\right) d u \\
& =\frac{(2 \pi)^{3 k / 2+1} n^{k / 2}}{\alpha^{k+1}} \cos \left(\frac{4 \pi^{2} n}{\alpha}-\frac{k \pi}{2}\right) \\
& =(-1)^{(k-1) / 2} \frac{(2 \pi)^{3 k / 2+1} n^{k / 2}}{\alpha^{k+1}} \sin \left(\frac{4 \pi^{2} n}{\alpha}\right) \\
& =(-1)^{(k-1) / 2} \frac{(2 \pi)^{3 k / 2+1} n^{k / 2}}{\alpha^{k+1}} \sin (\beta n),
\end{aligned}
$$

since $\alpha \beta=4 \pi^{2}$.

The preliminary details have been dispensed, and we are now ready to apply the Voronoï summation formula (6.3). Using the calculations (6.9) and (6.11) and the parameters defined above, we formally find that

$$
\begin{aligned}
\sum_{n=1}^{\infty} \sigma_{k}(n) \sin (\alpha n)= & \int_{0}^{\infty}\left(\frac{\zeta(1-k)}{2 \pi}+\frac{\zeta(k+1) t^{k}}{(2 \pi)^{k+1}}\right) \sin \left(\frac{\alpha t}{2 \pi}\right) d t \\
& -\left(\frac{2 \pi}{\alpha}\right)^{k+1} \sum_{n=1}^{\infty} \sigma_{k}(n) \sin (\beta n) .
\end{aligned}
$$

Thus, if we replace $s$ by $k$ in (2.17) and assume that $k$ is an odd integer, then (6.12) is as close as we can get in our efforts to formally derive (2.17). Note that on the right side of (6.12) a minus sign appears, in contrast to the right side of (2.17), and that a divergent integral appears on the right-hand side of (6.12), in place of the expressions involving the Riemann zeta function appearing in (2.17).

We now turn to (2.16), and once more we make an application of the Voronoï summation formula. Note that the series on the right-hand side of (2.16) does not converge for any real value of $s$, since $\sigma_{s}(n) \geq n^{s}$. Also note that for $x$ sufficiently large and for $\sigma>\frac{1}{2}$, each expression in (2.16) tends to 0 as $x$ tends to $\infty$, except for $-2 \pi \sqrt{\pi x} \zeta(-s) \tan \frac{1}{2} \pi s$, which tends to $\infty$.

To effect our application of Theorem 6.1 , we need the integral evaluation $[\mathbf{1 7}$, p. 709 , formula 6.565$, no. 2$]$

$$
\int_{0}^{\infty} x^{\nu+1} J_{\nu}(b x)\left(x^{2}+a^{2}\right)^{-\nu-1 / 2} d x=\frac{\sqrt{\pi} b^{\nu-1}}{2 e^{a b} \Gamma\left(\nu+\frac{1}{2}\right)},
$$

where Re $a>0, b>0, \operatorname{Re} \nu>-\frac{1}{2}$, and $J_{\nu}(x)$ denotes the ordinary Bessel function of order $\nu$.

Apply the Voronoï summation formula (6.3) twice, with $a=0, x=\infty$, and $f(t)=$ $(x \mp i t)^{-k-1 / 2}$, under the same conditions (6.6) and (6.7) as in our previous application. When $k$ 
is an odd positive integer, we are thus led to evaluate

$$
\begin{aligned}
\int_{0}^{\infty} t^{k / 2} & J_{k}\left(2 \sqrt{\mu_{n}} t\right)(x-i t)^{-k-1 / 2} d t \\
& =2 \int_{0}^{\infty} u^{k+1} J_{k}\left(2 u \sqrt{\mu_{n}}\right)\left(x-i u^{2}\right)^{-k-1 / 2} d u \\
& =2(-i)^{-k-1 / 2} \int_{0}^{\infty} u^{k+1} J_{k}\left(2 u \sqrt{\mu_{n}}\right)\left(u^{2}+i x\right)^{-k-1 / 2} d u \\
& =\frac{2(-i)^{-k-1 / 2} \sqrt{\pi}\left(2 \sqrt{\mu_{n}}\right)^{k-1}}{2^{k} \exp \left(2 \sqrt{\mu_{n} x} e^{\pi i / 4}\right) \Gamma\left(k+\frac{1}{2}\right)},
\end{aligned}
$$

where we have used (6.13) with $a=\sqrt{x} e^{\pi i / 4}, b=2 \sqrt{\mu_{n}}$, and $\nu=k$. Using (6.13) once again, but now with $a=\sqrt{x} e^{-\pi i / 4}$, we find by a similar calculation that

$$
\int_{0}^{\infty} t^{k / 2} J_{k}\left(2 \sqrt{\mu_{n} t}\right)(x+i t)^{-k-1 / 2} d t=\frac{2(-i)^{-k-1 / 2} \sqrt{\pi}\left(2 \sqrt{\mu_{n}}\right)^{k-1}}{2^{k} \exp \left(2 \sqrt{\mu_{n} x} e^{-\pi i / 4}\right) \Gamma\left(k+\frac{1}{2}\right)} .
$$

Employing (6.14) and (6.15), recalling (6.6) and (6.7), and remembering that $k$ is an odd positive integer, we find that the right-hand side of (6.3) becomes

$$
\begin{aligned}
& \frac{(-1)^{(k+1) / 2} \sqrt{\pi}}{\Gamma\left(k+\frac{1}{2}\right)} \sum_{n=1}^{\infty} \frac{\sigma_{k}(n)}{\sqrt{2 \pi n}}\left(e^{-2 \sqrt{2 \pi n x}(1+i) / \sqrt{2}+i \pi(k+1 / 2) / 2}\right. \\
& \left.-e^{-2 \sqrt{2 \pi n x}(1-i) / \sqrt{2}-i \pi(k+1 / 2) / 2}\right) \\
& =\frac{(-1)^{(k+1) / 2}}{\sqrt{2} \Gamma\left(k+\frac{1}{2}\right)} \sum_{n=1}^{\infty} \frac{\sigma_{k}(n)}{\sqrt{n}}\left(e^{-2 \sqrt{\pi n x}(1+i)} i(-1)^{(k-1) / 2} e^{\pi i / 4}\right. \\
& =-\frac{i}{\sqrt{2} \Gamma\left(k+\frac{1}{2}\right)} \sum_{n=1}^{\infty} \frac{\sigma_{k}(n)}{\sqrt{n}}\left(e^{-2 \sqrt{\pi n x}(1+i)} e^{\pi i / 4}+e^{-2 \sqrt{\pi n x}(1-i)} e^{-\pi i / 4}\right) \\
& =-\frac{i}{\sqrt{2} \Gamma\left(k+\frac{1}{2}\right)} \sum_{n=1}^{\infty} \frac{\sigma_{k}(n)}{\sqrt{n}} e^{-2 \sqrt{\pi n x}}\left(e^{-2 i \sqrt{\pi n x}+\pi i / 4}+e^{2 i \sqrt{\pi n x}-\pi i / 4}\right) \\
& =-\frac{i \sqrt{2}}{\Gamma\left(k+\frac{1}{2}\right)} \sum_{n=1}^{\infty} \frac{\sigma_{k}(n)}{\sqrt{n}} e^{-2 \sqrt{\pi n x}} \cos \left(2 \sqrt{\pi n x}-\frac{1}{4} \pi\right) \\
& =-\frac{i \sqrt{2}}{\Gamma\left(k+\frac{1}{2}\right)} \sum_{n=1}^{\infty} \frac{\sigma_{k}(n)}{\sqrt{n}} e^{-2 \sqrt{\pi n x}} \sin \left(2 \sqrt{\pi n x}+\frac{1}{4} \pi\right) .
\end{aligned}
$$

Finally, using the calculation above in (6.3) and employing also (6.9), we conclude that

$$
\begin{aligned}
\sum_{n=1}^{\infty} \sigma_{k}(n) & \left\{(x-i n)^{-k-1 / 2}-(x+i n)^{-k-1 / 2}\right\} \\
= & \int_{0}^{\infty}\left(\frac{\zeta(1-k)}{2 \pi}+\frac{\zeta(k+1) t^{k}}{(2 \pi)^{k+1}}\right)\left((x-i t)^{-k-1 / 2}-(x+i t)^{-k-1 / 2}\right) d t \\
& -\frac{i \sqrt{2}}{\Gamma\left(k+\frac{1}{2}\right)} \sum_{n=1}^{\infty} \frac{\sigma_{k}(n)}{\sqrt{n}} e^{-2 \sqrt{\pi n x}} \sin \left(2 \sqrt{\pi n x}+\frac{1}{4} \pi\right),
\end{aligned}
$$

which should be compared with (2.16). Observe that the integral on the right-hand side of (6.16) diverges, although it can be subdivided into two improper integrals, one of which converges and is elementary, and the other of which diverges. 


\section{Second Attempts to Find Meanings for Entries 2.4 and 2.3}

In this section, we use Mellin transforms in our attempts to find meaningful interpretations for Entries 2.4 and 2.3. We record some preliminary notation and results that are needed. The residue of a meromorphic function $f(z)$ at a pole $z=z_{0}$ is denoted by

$$
R_{z_{0}} f=R_{z_{0}}
$$

where the identification of the function $f$ is deleted if its definition is clear. For $-1<\operatorname{Re} z=c<1$, we need the Mellin transforms [16, p. 317, formula (1)]

$$
\begin{gathered}
\int_{0}^{\infty} \sin (a t) t^{z-1} d t=\Gamma(z) \sin \left(\frac{1}{2} \pi z\right) a^{-z}, \quad a>0 \\
\sin t=\frac{1}{2 \pi i} \int_{c-i \infty}^{c+i \infty} \Gamma(z) \sin \left(\frac{1}{2} \pi z\right) t^{-z} d z .
\end{gathered}
$$

For $x>0$ and $c=\operatorname{Re} z>0[\mathbf{1 6}$, p. 312, formula (1)],

$$
e^{-x}=\frac{1}{2 \pi i} \int_{c-i \infty}^{c+i \infty} \Gamma(z) x^{-z} d z
$$

When $s=\sigma+i t$, with $\sigma$ and $t$ both real, we need Stirling's formula for $\Gamma(s)$ in a vertical strip, namely $[\mathbf{1 7}$, p. 945 , formula 8.328 , no. 1$]$,

$$
|\Gamma(\sigma+i t)| \sim \sqrt{2 \pi}|t|^{\sigma-\frac{1}{2}} \exp \left(-\frac{1}{2} \pi|t|\right),
$$

as $|t| \rightarrow \infty$, uniformly in every vertical strip $\sigma_{1} \leq \sigma \leq \sigma_{2}$. The Riemann zeta function $\zeta(s)$ has the Laurent expansion [29, p. 16, Eq. (2.1.16)]

$$
\zeta(s)=\frac{1}{s-1}+\gamma+\cdots,
$$

where $\gamma$ denotes Euler's constant. For the convergence of certain integrals, we need elementary upper bounds for the growth of $\zeta(s)$ in a vertical strip. Thus, the estimates [29, p. 81]

$$
\zeta(\sigma+i t)=O\left(t^{\frac{1}{2}-\sigma}\right), \quad \sigma<-\delta<0 ; \quad \zeta(\sigma+i t)=O\left(t^{\frac{3}{2}+\delta}\right), \quad \sigma>-\delta
$$

will suffice.

We examine (2.17) first and emphasize that our goal is to replace the divergent series that appear in this formula by convergent series or integrals. Thus, our procedures will be formal.

Using (7.2) and (6.4), we find that

$$
\begin{aligned}
\sum_{n=1}^{\infty} \sigma_{s}(n) \sin (n \alpha) & =\frac{1}{2 \pi i} \sum_{n=1}^{\infty} \sigma_{s}(n) \int_{c-i \infty}^{c+i \infty} \Gamma(z) \sin \left(\frac{1}{2} \pi z\right)(n \alpha)^{-z} d z \\
& =\frac{1}{2 \pi i} \int_{c-i \infty}^{c+i \infty} \Gamma(z) \sin \left(\frac{1}{2} \pi z\right) \alpha^{-z} \sum_{n=1}^{\infty} \frac{\sigma_{s}(n)}{n^{z}} d z \\
& =\frac{1}{2 \pi i} \int_{c-i \infty}^{c+i \infty} \Gamma(z) \sin \left(\frac{1}{2} \pi z\right) \alpha^{-z} \zeta(z-s) \zeta(z) d z=: I,
\end{aligned}
$$

where $-1<c=\operatorname{Re} z<1$ and $c \neq 1+\operatorname{Re} s$. Let $u:=1-z$. Then, by the functional equation (2.20) for $\zeta(s)$ and (7.7),

$$
\begin{aligned}
I= & \frac{1}{2 \pi i} \int_{1-c-i \infty}^{1-c+i \infty} \Gamma(1-u) \cos \left(\frac{1}{2} \pi u\right) \alpha^{u-1} \zeta(1-(u+s)) \zeta(1-u) d u \\
= & \frac{1}{2 \pi i} \int_{1-c-i \infty}^{1-c+i \infty} \Gamma(1-u) \cos \left(\frac{1}{2} \pi u\right) \alpha^{u-1} 2(2 \pi)^{-u-s} \cos \left(\frac{\pi}{2}(u+s)\right) \Gamma(u+s) \zeta(u+s) \\
& \times 2(2 \pi)^{-u} \cos \left(\frac{1}{2} \pi u\right) \Gamma(u) \zeta(u) d u .
\end{aligned}
$$

Applying the two identities

$$
\Gamma(u) \Gamma(1-u) \cos ^{2}\left(\frac{1}{2} \pi u\right)=\frac{\pi}{2} \cot \left(\frac{1}{2} \pi u\right),
$$




$$
\cot \left(\frac{1}{2} \pi u\right) \cos \left(\frac{1}{2} \pi(u+s)\right)=\cos \left(\frac{1}{2} \pi s\right) \csc \left(\frac{1}{2} \pi u\right)-\sin \left(\frac{1}{2} \pi(u+s)\right)
$$

in (7.8), we conclude that

$$
I=J-K
$$

where

$$
J:=\frac{1}{2 \pi i} \int_{1-c-i \infty}^{1-c+i \infty}(2 \pi)^{1-2 u-s} \alpha^{u-1} \cos \left(\frac{1}{2} \pi s\right) \csc \left(\frac{1}{2} \pi u\right) \Gamma(u+s) \zeta(u+s) \zeta(u) d u
$$

and

$$
\begin{aligned}
K & :=\frac{1}{2 \pi i} \int_{1-c-i \infty}^{1-c+i \infty}(2 \pi)^{1-2 u-s} \alpha^{u-1} \sin \left(\frac{1}{2} \pi(u+s)\right) \Gamma(u+s) \zeta(u+s) \zeta(u) d u \\
& =\alpha^{-(s+1) / 2} \beta^{(s+1) / 2} \sum_{n=1}^{\infty} \sigma_{s}(n) \frac{1}{2 \pi i} \int_{1-c-i \infty}^{1-c+i \infty} \sin \left(\frac{1}{2} \pi(u+s)\right) \Gamma(u+s)(\beta n)^{-u-s} d u
\end{aligned}
$$

since $4 \pi^{2}=\alpha \beta$. From (7.4) and (7.6), we see that $J$ converges while $K$ diverges. We consider two cases.

CASE 1: Assume that $-1<c<0,-1<\operatorname{Re} s<2$, and $c<\operatorname{Re} s$. First, we examine $J$. Let $-\operatorname{Re} s-1<d<-\operatorname{Re} s$. If we move the line of integration from $u=1-c$ to $u=d$, then the poles of the integrand of $J$ lying between these two lines are $u=1,1-s, 0,-s$. Thus, using (7.4) and (7.6) to justify shifting the line of integration, we find, from (7.12), that

$$
J=R_{1}+R_{1-s}+R_{0}+R_{-s}+J_{1},
$$

where

$$
J_{1}:=\frac{1}{2 \pi i} \int_{d-i \infty}^{d+i \infty}(2 \pi)^{1-2 u-s} \alpha^{u-1} \cos \left(\frac{1}{2} \pi s\right) \csc \left(\frac{1}{2} \pi u\right) \Gamma(u+s) \zeta(u+s) \zeta(u) d u .
$$

Since, by (6.8), (2.20), and (7.5),

$$
\begin{aligned}
R_{1} & =-\frac{1}{2} \zeta(-s) \cot \left(\frac{1}{2} \pi s\right), \\
R_{1-s} & =(2 \pi)^{s-1} \alpha^{-s} \zeta(1-s), \\
R_{0} & =-\alpha^{-1} \zeta(1-s), \\
R_{-s} & =\frac{1}{2}(2 \pi)^{1+s} \alpha^{-s-1} \zeta(-s) \cot \left(\frac{1}{2} \pi s\right),
\end{aligned}
$$

we find from (7.14) that

$$
\begin{aligned}
J= & -\frac{1}{2} \zeta(-s) \cot \left(\frac{1}{2} \pi s\right)+(2 \pi)^{s-1} \alpha^{-s} \zeta(1-s)-\alpha^{-1} \zeta(1-s) \\
& +\frac{1}{2}(2 \pi)^{1+s} \alpha^{-s-1} \zeta(-s) \cot \left(\frac{1}{2} \pi s\right)+J_{1} .
\end{aligned}
$$

Using (7.19) and (7.13) in (7.11), multiplying both sides of (7.11) by $\alpha^{(s+1) / 2}$, and once again using the condition $\alpha \beta=4 \pi^{2}$, we conclude that

$$
\begin{aligned}
& \alpha^{(s+1) / 2}\left\{\frac{1}{\alpha} \zeta(1-s)+\frac{1}{2} \zeta(-s) \cot \left(\frac{1}{2} \pi s\right)+\sum_{n=1}^{\infty} \sigma_{s}(n) \sin (n \alpha)\right\} \\
& =\beta^{(s+1) / 2}\left\{\frac{1}{\beta} \zeta(1-s)+\frac{1}{2} \zeta(-s) \cot \left(\frac{1}{2} \pi s\right)\right\}+\alpha^{(s+1) / 2}\left(J_{1}-K\right),
\end{aligned}
$$

where $K$ is defined by (7.13).

CASE 2: Now assume that $0<c<1,-1<\operatorname{Re} s<1$, and $\operatorname{Re} s<c<\operatorname{Re} s+1$. Let $b_{1}$ be a real number with $-1<b_{1}<0$ and $-1<b_{1}<-\operatorname{Re} s$. Then, the poles of the integrand of $J$ that exist 
between the two lines $u=1-c$ and $u=b_{1}$ are $u=-s, 0$. Thus, shifting the line of integration with the aid of (7.4) and (7.6), and using (7.17) and (7.18), we find that

$$
\begin{aligned}
J & =R_{0}+R_{-s}+J_{2} \\
& =-\alpha^{-1} \zeta(1-s)+\frac{1}{2}(2 \pi)^{1+s} \alpha^{-s-1} \zeta(-s) \cot \left(\frac{1}{2} \pi s\right)+J_{2},
\end{aligned}
$$

where

$$
J_{2}:=\frac{1}{2 \pi i} \int_{b_{1}-i \infty}^{b_{1}+i \infty}(2 \pi)^{1-2 u-s} \alpha^{u-1} \cos \left(\frac{1}{2} \pi s\right) \csc \left(\frac{1}{2} \pi u\right) \Gamma(u+s) \zeta(u+s) \zeta(u) d u .
$$

Let $b_{2}$ be a real number with $1<b_{2}<2$ and $1-\operatorname{Re} s<b_{2}<2$. Then, the poles of the integrand of $J$ that exist between the two lines $u=1-c$ and $u=b_{2}$ are $u=1,1-s$. Thus, shifting the line of integration with the aid of (7.4) and (7.6), and using (7.15) and (7.16), we find that

$$
\begin{aligned}
J & =-\left(R_{1}+R_{1-s}\right)+J_{3} \\
& =\frac{1}{2} \zeta(-s) \cot \left(\frac{1}{2} \pi s\right)-(2 \pi)^{s-1} \alpha^{-s} \zeta(1-s)+J_{3},
\end{aligned}
$$

where

$$
J_{3}:=\frac{1}{2 \pi i} \int_{b_{2}-i \infty}^{b_{2}+i \infty}(2 \pi)^{1-2 u-s} \alpha^{u-1} \cos \left(\frac{1}{2} \pi s\right) \csc \left(\frac{1}{2} \pi u\right) \Gamma(u+s) \zeta(u+s) \zeta(u) d u .
$$

Combining the two representations (7.21) and (7.22) of $J$, we deduce that

$$
\begin{aligned}
2 J= & -\alpha^{-1} \zeta(1-s)+\frac{1}{2}(2 \pi)^{1+s} \alpha^{-s-1} \zeta(-s) \cot \left(\frac{1}{2} \pi s\right) \\
& +\frac{1}{2} \zeta(-s) \cot \left(\frac{1}{2} \pi s\right)-(2 \pi)^{s-1} \alpha^{-s} \zeta(1-s)+J_{2}+J_{3} .
\end{aligned}
$$

Since $-1<\operatorname{Re}(u+s)<1$, we can use $(7.7)$ in $(7.13)$ to deduce that

$$
K=\alpha^{-(s+1) / 2} \beta^{(s+1) / 2} \sum_{n=1}^{\infty} \sigma_{s}(n) \sin (\beta n) .
$$

Now return to (7.11), multiply both sides of (7.11) by $2 \alpha^{(s+1) / 2}$, and use (7.23) and (7.24) in (7.11) to conclude that

$$
\begin{aligned}
& \alpha^{(s+1) / 2}\left\{\frac{1}{\alpha} \zeta(1-s)-\frac{1}{2} \zeta(-s) \cot \left(\frac{1}{2} \pi s\right)+2 \sum_{n=1}^{\infty} \sigma_{s}(n) \sin (n \alpha)\right\} \\
& =\beta^{(s+1) / 2}\left\{-\frac{1}{\beta} \zeta(1-s)+\frac{1}{2} \zeta(-s) \cot \left(\frac{1}{2} \pi s\right)-2 \sum_{n=1}^{\infty} \sigma_{s}(n) \sin (\beta n)\right\} \\
& +\alpha^{(s+1) / 2}\left(J_{2}+J_{3}\right) .
\end{aligned}
$$

In summary, we observe that both (7.20) and (7.25) are "very close" to (2.17). Unfortunately, the signs of the divergent series on the right-hand sides of (7.20) and (7.25) do not agree with that in (2.17). It is also unfortunate that Mellin transforms appear in both (7.20) and (7.25), although, on the other hand, the integrals are indeed convergent.

Next, we examine Entry 2.4. By (7.3), (7.2), and (6.4), for $c>0, c-\frac{1}{2}<\operatorname{Re} s<c+\frac{3}{2}$, and $x>0$,

$$
\begin{aligned}
& \Gamma\left(s+\frac{1}{2}\right) \sum_{n=1}^{\infty} \frac{\sigma_{s}(n)}{2 i}\left\{(x-i n)^{-s-\frac{1}{2}}-(x+i n)^{-s-\frac{1}{2}}\right\} \\
& =\sum_{n=1}^{\infty} \sigma_{s}(n) \int_{0}^{\infty} e^{-x t} \sin (n t) t^{s-\frac{1}{2}} d t \\
& =\sum_{n=1}^{\infty} \sigma_{s}(n) \int_{0}^{\infty} \frac{1}{2 \pi i} \int_{c-i \infty}^{c+i \infty} \Gamma(z)(x t)^{-z} d z \sin (n t) t^{s-\frac{1}{2}} d t
\end{aligned}
$$




$$
\begin{aligned}
= & \sum_{n=1}^{\infty} \sigma_{s}(n) \frac{1}{2 \pi i} \int_{c-i \infty}^{c+i \infty} \Gamma(z) x^{-z} \int_{0}^{\infty} \sin (n t) t^{-z+s-\frac{1}{2}} d t d z \\
= & \sum_{n=1}^{\infty} \sigma_{s}(n) \frac{1}{2 \pi i} \int_{c-i \infty}^{c+i \infty} \Gamma(z) x^{-z} \Gamma\left(s-z+\frac{1}{2}\right) \sin \left(\frac{1}{2} \pi\left(s-z+\frac{1}{2}\right)\right) n^{-s+z-\frac{1}{2}} d z \\
= & \frac{1}{2 \pi i} \int_{c-i \infty}^{c+i \infty} \Gamma(z) x^{-z} \Gamma\left(s-z+\frac{1}{2}\right) \sin \left(\frac{1}{2} \pi\left(s-z+\frac{1}{2}\right)\right) \\
& \times \zeta\left(\frac{1}{2}-z\right) \zeta\left(s-z+\frac{1}{2}\right) d z \\
= & : \mathcal{I} .
\end{aligned}
$$

Let $v:=s-z+\frac{1}{2}$. Then

$$
\mathcal{I}=\frac{1}{2 \pi i} \int_{\operatorname{Re} s+\frac{1}{2}-c-i \infty}^{\operatorname{Re} s+\frac{1}{2}-c+i \infty} \Gamma(v) \Gamma\left(s-v+\frac{1}{2}\right) \sin \left(\frac{1}{2} \pi v\right) x^{v-s-\frac{1}{2}} \zeta(v-s) \zeta(v) d v .
$$

Next, let $w:=1-v$. Then, using (2.20), (7.9), and (7.10) and arguing as we did above in the "proof" of Entry 2.4, we find that

$$
\begin{aligned}
\mathcal{I}= & \frac{1}{2 \pi i} \int_{c-\operatorname{Re} s+\frac{1}{2}-i \infty}^{c-\operatorname{Re} s+\frac{1}{2}+i \infty} \Gamma(1-w) \Gamma\left(s+w-\frac{1}{2}\right) \cos \left(\frac{1}{2} \pi w\right) x^{-w-s+\frac{1}{2}} \\
& \times \zeta(1-(w+s)) \zeta(1-w) d w \\
= & \frac{1}{2 \pi i} \int_{c-\operatorname{Re} s+\frac{1}{2}-i \infty}^{c-\operatorname{Re} s+\frac{1}{2}+i \infty} \Gamma(1-w) \Gamma\left(s+w-\frac{1}{2}\right) \Gamma(w) \Gamma(w+s) \cos ^{2}\left(\frac{1}{2} \pi w\right) \\
& \times \cos \left(\frac{1}{2} \pi(w+s)\right) 4(2 \pi)^{-2 u-s} x^{-w-s+\frac{1}{2}} \zeta(w+s) \zeta(w) d w \\
= & \mathfrak{J}-\mathfrak{K},
\end{aligned}
$$

where

$$
\begin{aligned}
\mathfrak{J}:= & \frac{\cos \left(\frac{1}{2} \pi s\right)}{2 \pi i} \int_{c-\operatorname{Re} s+\frac{1}{2}-i \infty}^{c-\operatorname{Re} s+\frac{1}{2}+i \infty} \Gamma\left(s+w-\frac{1}{2}\right) \Gamma(w+s) \csc \left(\frac{1}{2} \pi w\right) x^{-w-s+\frac{1}{2}}(2 \pi)^{1-2 w-s} \\
& \times \zeta(w+s) \zeta(w) d w \\
\mathfrak{K}:= & \frac{1}{2 \pi i} \int_{c-\operatorname{Re} s+\frac{1}{2}-i \infty}^{c-\operatorname{Re} s+\frac{1}{2}+i \infty} \Gamma\left(s+w-\frac{1}{2}\right) \Gamma(w+s) \sin \left(\frac{1}{2} \pi(w+s)\right) x^{-w-s+\frac{1}{2}} \\
& \times(2 \pi)^{1-2 w-s} \zeta(w+s) \zeta(w) d w .
\end{aligned}
$$

Generally, we assume that $-2<\operatorname{Re} s<2$ and that $0<c<\frac{1}{2}$. Assume also that $0<$ $c+\frac{1}{2}-\operatorname{Re} s$. In moving the line of integration twice below, we need to calculate the residues of the integrand of $\mathfrak{J}$ at the poles $w=\frac{1}{2}-s, 0,-s, 1-s, 1$. Using the value $\Gamma\left(\frac{1}{2}\right)=\sqrt{\pi},(6.8)$, (2.20), and formally writing $\zeta\left(\frac{1}{2}\right) \zeta\left(\frac{1}{2}-s\right)=\sum_{n=1}^{\infty} \sigma_{s}(n) / \sqrt{n}$, we find that

$$
\begin{aligned}
R_{\frac{1}{2}-s} & =\sqrt{\frac{\pi}{2}}(2 \pi)^{s}\left(1+\tan \left(\frac{\pi}{2}\left(\frac{1}{2}+s\right)\right)\right) \sum_{n=1}^{\infty} \frac{\sigma_{s}(n)}{\sqrt{n}}, \\
R_{0} & =-\frac{\Gamma\left(s+\frac{1}{2}\right)}{\left(s-\frac{1}{2}\right) x^{s-\frac{1}{2}}} \zeta(1-s), \\
R_{-s} & =-(2 \pi)^{s} 2 \pi \sqrt{\pi x} \zeta(-s) \cot \left(\frac{1}{2} \pi s\right), \\
R_{1-s} & =(2 \pi)^{s} \frac{\zeta(1-s)}{2 \sqrt{\pi x}}
\end{aligned}
$$




$$
R_{1}=-\frac{\Gamma\left(s+\frac{1}{2}\right)}{2 x^{s+\frac{1}{2}}} \zeta(-s) \cot \left(\frac{1}{2} \pi s\right) .
$$

Let $r_{1}$ be a real number with $r_{1}<-\operatorname{Re} s$ and $-2<r_{1}<0$. We first move the line of integration from $w=c-\operatorname{Re} s-\frac{1}{2}$ to $w=r_{1}$ and use (7.31) and (7.32) to find that

$$
\begin{aligned}
\mathfrak{J}= & R_{0}+R_{-s}+R_{\frac{1}{2}-s}+\mathfrak{J}_{1} \\
= & \frac{\Gamma\left(s+\frac{1}{2}\right)}{\left(s-\frac{1}{2}\right) x^{s-\frac{1}{2}}} \zeta(1-s)+(2 \pi)^{s} 2 \pi \sqrt{\pi x} \zeta(-s) \cot \left(\frac{1}{2} \pi s\right) \\
& +\sqrt{\frac{\pi}{2}}(2 \pi)^{s}\left(1+\tan \left(\frac{\pi}{2}\left(\frac{1}{2}+s\right)\right)\right) \sum_{n=1}^{\infty} \frac{\sigma_{s}(n)}{\sqrt{n}}+\mathfrak{J}_{1},
\end{aligned}
$$

where

$$
\begin{aligned}
\mathfrak{J}_{1}:= & \cos \left(\frac{1}{2} \pi s\right)(2 \pi)^{s} \sum_{n=1}^{\infty} \frac{\sigma_{s}(n)}{\sqrt{n}} \frac{1}{2 \pi i} \int_{r_{1}-i \infty}^{r_{1}+i \infty}\left(4 \pi^{2} n x\right)^{-w-s+\frac{1}{2}} \Gamma\left(s+w-\frac{1}{2}\right) \\
& \times \Gamma(w+s) \csc \left(\frac{1}{2} \pi w\right) d w .
\end{aligned}
$$

Secondly, let $k_{1}$ be a real number with $1-\operatorname{Re} s<k_{1}$ and $1<k_{1}<2$. If we move the line of integration of $\mathfrak{K}$ to $w=k_{1}$, observe that the residues of the integrand of $\mathfrak{K}$ at the poles $w=1-s, 1$ are the same as those of the integrand of $\mathfrak{J}$, and use the residues (7.33), (7.30), and (7.34), we deduce that

$$
\begin{aligned}
\mathfrak{K} & =\mathfrak{K}_{1}-\left(R_{1-s}+R_{1}\right) \\
& =\mathfrak{K}_{1}-(2 \pi)^{s} \frac{\zeta(1-s)}{2 \sqrt{\pi x}}+\frac{\Gamma\left(s+\frac{1}{2}\right)}{2 x^{s+\frac{1}{2}}} \zeta(-s) \cot \left(\frac{1}{2} \pi s\right),
\end{aligned}
$$

where

$$
\mathfrak{K}_{1}:=\sum_{n=1}^{\infty} \frac{\sigma_{s}(n)}{\sqrt{n}} \frac{1}{2 \pi i} \int_{k_{1}-i \infty}^{k_{1}+i \infty}\left(4 \pi^{2} n x\right)^{-w-s+\frac{1}{2}} \Gamma\left(s+w-\frac{1}{2}\right) \Gamma(w+s) \sin \left(\frac{1}{2} \pi(w+s)\right) d w
$$

by (6.4). Finally, using (7.26), (7.27), (7.28), (7.29), (7.35), and (7.36), we conclude that

$$
\begin{aligned}
& \Gamma\left(s+\frac{1}{2}\right)\left\{\frac{\zeta(1-s)}{\left(s-\frac{1}{2}\right) x^{s-\frac{1}{2}}}+\frac{\zeta(-s)}{2 x^{s+\frac{1}{2}}} \cot \left(\frac{1}{2} \pi s\right)\right. \\
& \left.+\sum_{n=1}^{\infty} \frac{\sigma_{s}(n)}{2 i}\left\{(x-i n)^{-s-\frac{1}{2}}-(x+i n)^{-s-\frac{1}{2}}\right\}\right\} \\
& =(2 \pi)^{s}\left\{\frac{\zeta(1-s)}{2 \sqrt{\pi x}}-2 \pi \sqrt{\pi x} \zeta(-s) \cot \left(\frac{1}{2} \pi s\right)\right\} \\
& +\sqrt{\frac{\pi}{2}}(2 \pi)^{s}\left(1+\tan \left(\frac{\pi}{2}\left(\frac{1}{2}+s\right)\right)\right) \sum_{n=1}^{\infty} \frac{\sigma_{s}(n)}{\sqrt{n}}+\mathfrak{J}_{1}-\mathfrak{K}_{1} .
\end{aligned}
$$

Entry 2.3 should now be compared with (7.37). At the beginning of our derivation of (7.37), we imposed conditions on $c$ and Re $s$. We could impose different restrictions on $c$ and $\operatorname{Re} s$ and derive formulas similar to (7.37), but we refrain from doing so here. For example, if $c>\frac{1}{2}$ and $0<c+\frac{1}{2}-\operatorname{Re} s<1$, then we can deduce the same result (7.37) once again.

\section{Further Questionable Claims in the Lost Notebook}

Recall the familiar notation from the theory of $q$-series. Let $(a ; q)_{0}:=1$. If $n$ is a positive integer, set

$$
(a ; q)_{n}:=(1-a)(1-a q) \cdots\left(1-a q^{n-1}\right), \quad(a ; q)_{\infty}=\lim _{n \rightarrow \infty}(a ; q)_{n}, \quad|q|<1 .
$$


On page 4 in his lost notebook [27], Ramanujan offers three formulas containing divergent $q$-series. We record two of them.

ENTRY 8.1. If $a \neq 0$,

$$
\sum_{n=0}^{\infty} \frac{(-1)^{n}\left(q ; q^{2}\right)_{n} q^{n^{2}}}{\left(-a q^{2} ; q^{2}\right)_{n}\left(-q^{2} / a ; q^{2}\right)_{n}}=\sum_{n=0}^{\infty} \frac{(-1)^{n}\left(q ; q^{2}\right)_{n}}{(-a q ; q)_{n}(-q / a ; q)_{n}}+\frac{\left(q ; q^{2}\right)_{\infty} \varphi(-q)}{2(-a q ; q)_{\infty}(-q / a ; q)_{\infty}} .
$$

Since the first series on the right-hand side of (8.2) is divergent, the proposed identity has no meaning at present. However, as was noted in [1, p. 37],

$$
\lim _{\alpha \rightarrow 1^{-}} \sum_{n=0}^{\infty} \frac{(-1)^{n}(\alpha q ; q)_{n}\left(q ; q^{2}\right)_{n}}{(q ; q)_{n}(-\alpha a q ; q)_{n}(-\alpha q / a ; q)_{n}} \alpha^{n}=\frac{\left(q ; q^{2}\right)_{\infty}}{2\left(q^{2} ; q^{2}\right)_{\infty}} \sum_{n=-\infty}^{\infty} \frac{(1+1 / a)(1+a) q^{n(n+1) / 2}}{\left(1+a q^{n}\right)\left(1+q^{n} / a\right)}
$$

which follows from Watson's q-analogue of Whipple's theorem [2, p. 266]. With the replacement of the aforementioned divergent series by the right-hand side of (8.3), then (8.2) becomes a valid identity [2, Entry 12.3.4, p. 267]; see [2, pp. 267-268] for a proof.

Appearing in the last entry on page 4 of Ramanujan's lost notebook is Ramanujan's sixth order mock theta function

$$
\psi(q):=\sum_{n=1}^{\infty} \frac{(-1)^{n-1} q^{n^{2}}\left(q ; q^{2}\right)_{n-1}}{(-q ; q)_{2 n-1}} .
$$

ENTRY 8.2. We have

$$
\sum_{n=0}^{\infty} \frac{(-1)^{n}\left(q^{3} ; q^{6}\right)_{n}}{\left(-q^{3} ; q^{3}\right)_{n}^{2}}-2 \psi(q)=\frac{\varphi^{2}(-q)}{2 \Psi\left(q^{3}\right)}
$$

where

$$
\varphi(q):=\sum_{n=-\infty}^{\infty} q^{n^{2}} \quad \text { and } \quad \Psi(q):=\sum_{n=0}^{\infty} q^{n(n+1) / 2} .
$$

Since the series on the left-hand side of (8.4) does not converge, at present, this claim is also meaningless. We provide a similar meaningful interpretation of this questionable entry.

Putting $a=1$ and replacing $q$ by $q^{3}$ in (8.3), we can show that

$$
\sum_{n=0}^{\infty} \frac{(-1)^{n}\left(q^{3} ; q^{6}\right)_{n}}{\left(-q^{3} ; q^{3}\right)_{n}^{2}}=\lim _{\alpha \rightarrow 1^{-}} \sum_{n=0}^{\infty} \frac{(-1)^{n}\left(\alpha q^{3} ; q^{3}\right)_{n}\left(q^{3} ; q^{6}\right)_{n}}{\left(q^{3} ; q^{3}\right)_{n}\left(-\alpha q^{3} ; q^{3}\right)_{n}^{2}} \alpha^{n}=\frac{1}{\Psi\left(q^{3}\right)} \sum_{n=-\infty}^{\infty} \frac{q^{3 n(n+1) / 2}}{1+q^{3 n}}
$$

thus allowing (8.4) to be expressed in a correct form. Complete details may be found in the paper by Berndt and S. H. Chan [13].

Certainly, Ramanujan was aware that the divergent series in (8.1) and (8.4) are indeed divergent. We cannot ascertain if our interpretations of his divergent series are what Ramanujan had in mind, but since our versions do yield correct identities, we think that our interpretations are those intended by Ramanujan. Furthermore, as noted in [2], the corrected version of (8.1) resembles another identity on page 8 in Ramanujan's lost notebook. Also, the proof of (8.5) is relatively simple. We are unaware of a statement of Watson's $q$-analogue of Whipple's theorem in Ramanujan's writings. However, he doubtless knew of it or, at least, of several corollaries or special cases of it. See, for example, Entry 7 of Chapter 15 in Ramanujan's second notebook [26], $[\mathbf{9}$, p. 16], where a four-parameter corollary of Watson's theorem is stated.

We lastly consider an entry, where, indeed, Ramanujan did make a mistake. Published with Ramanujan's lost notebook [27, pp. 318-321] is a four-page, handwritten fragment on infinite series. Partial fraction expansions, the Riemann zeta-function $\zeta(s)$, divisor sums, Bernoulli numbers, and Euler numbers are featured in the formulas in this four-page manuscript. On page 318, prior to the incorrect entry, Ramanujan writes, "By the theory of residues it can be shown that". We think that Ramanujan implied that he used the calculus of residues to calculate the partial fraction decomposition that followed. Indeed, a formal application of the residue theorem would give the partial fraction decomposition that he claimed. However, for his deduction to be correct, he would need to apply the Mittag-Leffler Theorem, which unfortunately cannot be applied in 
this situation! We first state the incorrect expansion and then offer a correct version. Ramanujan used $n$ to denote a complex variable; we replace it with a more natural notation $w$.

ENTRY 8.3 (p. 318, formula (21)). If $\alpha$ and $\beta$ are positive numbers such that $\alpha \beta=\pi^{2}$, then

$$
\frac{\pi}{2} \cot (\sqrt{w \alpha}) \operatorname{coth}(\sqrt{w \beta})=\frac{1}{2 w}+\sum_{m=1}^{\infty}\left\{\frac{m \alpha \operatorname{coth}(m \alpha)}{w+m^{2} \alpha}+\frac{m \beta \operatorname{coth}(m \beta)}{w-m^{2} \beta}\right\} .
$$

ENTRY 8.4 (Corrected Version of (21)). Under the hypotheses of Entry 8.3,

$$
\frac{\pi}{2} \cot (\sqrt{w \alpha}) \operatorname{coth}(\sqrt{w \beta})=\frac{1}{2 w}+\frac{1}{2} \log \frac{\beta}{\alpha}+\sum_{m=1}^{\infty}\left\{\frac{m \alpha \operatorname{coth}(m \alpha)}{w+m^{2} \alpha}+\frac{m \beta \operatorname{coth}(m \beta)}{w-m^{2} \beta}\right\} .
$$

Thus, Ramanujan's claim (21) was correct except for the missing term $\frac{1}{2} \log \frac{\beta}{\alpha}$.

After his claim (21), Ramanujan recorded several corollaries arising from his (incorrect) partial fraction decomposition. All of the corollaries are indeed correct, but two of them do not follow from his partial fraction expansion; they do follow from the corrected version (8.7). Ramanujan undoubtedly had been familiar with these corollaries and almost certainly had derived them by other methods. Realizing that these results should be easy deductions of his expansion, he incorrectly assumed that they could be so derived and evidently did not work out the details. It is interesting that the same incorrect partial fraction expansion occurs in Entry 19(i) of Chapter 14 of his second notebook [26], [8, p. 271], where it was derived by a different method, namely a general elementary theorem, Entry 18 of Chapter 14 [26], [8, pp. 267-268]. For a detailed discussion of this four-page manuscript, see [10].

We have concentrated on some questionable formulas for which the corrections (if possible) or the proper interpretations have yet to be made. Furthermore, some formulas in the lost notebook contain errors that are a bit more serious than mere misprints, but which we have been able to correct. For example, on page 56, S.-Y. Kang [23, pp. 103-104, Theorem 4.4], [2, p. 37, Entry 1.8.4] discovered that a factor of $\{(1-k) /(1+k)\}^{1 / 10}$ is missing from one formula. On pages 210 and 212, Ramanujan recorded tables of values of the Rogers-Ramanujan continued fraction [12], [2, Chapter 2] and a cubic analogue of the classical Ramanujan-Weber class invariant [11], [3, Chapter 9], respectively. Most of the values in each table are missing, but it is clear that Ramanujan could have completed the tables; he was just running out of time, and there were other theorems to record and establish before he died. Ramanujan's widow, Janaki, told one of the present authors (BCB) in 1987 that her husband worked on mathematics, or in her words, he was "doing his sums," up until four days before he died, when "the pain was too great." With increasing pain and diminishing strength, Ramanujan can be forgiven for leaving us some incomplete or questionable formulas before he died.

\section{References}

[1] G. E. Andrews, Mordell integrals and Ramanujan's "lost" notebook, in Analytic Number Theory, M. I. Knopp, ed., Springer-Verlag Lecture Notes, No. 899, Springer-Verlag, Berlin, 1981, pp. 10-48.

[2] G. E. Andrews and B. C. Berndt, Ramanujan's Lost Notebook, Part I, Springer, New York, 2005.

[3] G. E. Andrews and B. C. Berndt, Ramanujan's Lost Notebook, Part II, Springer, New York, to appear.

[4] B. C. Berndt, Identities involving the coefficients of a class of Dirichlet series. I., Trans. Amer. Math. Soc. 137 (1969), 345-359.

[5] B. C. Berndt, Identities involving the coefficients of a class of Dirichlet series. III., Trans. Amer. Math. Soc. 146 (1969), 323-348.

[6] B. C. Berndt, Identities involving the coefficients of a class of Dirichlet series. V., Trans. Amer. Math. Soc. 160 (1971), 139-156.

[7] B. C. Berndt, Generalized Dedekind eta-functions and generalized Dedekind sums, Trans. Amer. Math. Soc. 178 (1973), 495-508.

[8] B. C. Berndt, Ramanujan's Notebooks, Part II, Springer-Verlag, New York, 1989.

[9] B. C. Berndt, Ramanujan's Notebooks, Part III, Springer-Verlag, New York, 1991.

[10] B. C. Berndt, An unpublished manuscript of Ramanujan on infinite series identities, J. Ramanujan Math. Soc. 19 (2004), 57-74.

[11] B. C. Berndt, H. H. Chan, S.-Y. Kang, and L.-C. Zhang, A certain quotient of eta-functions found in Ramanujan's lost notebook, Pacific J. Math. 202 (2002), 267-304. 
[12] B. C. Berndt, H. H. Chan, and L.-C. Zhang, Explicit evaluations of the Rogers-Ramanujan continued fraction, J. Reine Angew. Math. 480 (1996), 141-159.

[13] B. C. Berndt and S. H. Chan, Sixth order mock theta functions, Adv. Math. 216 (2007), 771-786.

[14] B. C. Berndt and A. Zaharescu, Weighted divisor sums and Bessel function series, Math. Ann. 335 (2006), 249-283.

[15] K. Chandrasekharan and R. Narasimhan, Hecke's functional equation and arithmetical identities, Ann. Math. (2) 74 (1961), 1-23.

[16] A. Erdélyi, ed., Tables of Integral Transforms, Vol. 1, McGraw-Hill, New York, 1954.

[17] I. S. Gradshteyn and I. M. Ryzhik, eds., Table of Integrals, Series, and Products, 5th ed., Academic Press, San Diego, 1994.

[18] G. H. Hardy, Some multiple integrals, Quart. J. Math. (Oxford) 39 (1908), 357-375.

[19] G. H. Hardy, On the expression of a number as the sum of two squares, Quart. J. Math. (Oxford) 46 (1915), 263-283.

[20] G. H. Hardy, On Dirichlet's divisor problem, Proc. London Math. Soc. (2) 15 (1916), 1-25.

[21] G. H. Hardy, Collected Papers, Vol. II, Oxford University Press, Oxford, 1967.

[22] G. H. Hardy, Collected Papers, Vol. V, Oxford University Press, Oxford, 1972.

[23] S.-Y. Kang, Some theorems on the Rogers-Ramanujan continued fraction and associated theta function identities in Ramanujan's lost notebook, Ramanujan J. 3 (1999), 91-111.

[24] A. P. Prudnikov, Yu. A. Brychkov, and O. I. Marichev, Integrals and Series, Vol. 2: Special Functions, Gordon and Breach, New York, 1986.

[25] H. Rademacher, Lectures on Elementary Number Theory, Blaisdell, New York, 1964.

[26] S. Ramanujan, Notebooks (2 volumes), Tata Institute of Fundamental Research, Bombay, 1957.

[27] S. Ramanujan, The Lost Notebook and Other Unpublished Papers, Narosa, New Delhi, 1988.

[28] E. C. Titchmarsh, The Theory of Functions, 2nd ed., Oxford University Press, Oxford, 1939.

[29] E. C. Titchmarsh, The Theory of the Riemann Zeta-function, Clarendon Press, Oxford, 1951.

[30] M. G. Voronoï, Sur une fonction transcendante et ses applications à la sommation de quelques séries, Ann. École Norm. Sup. (3) 21 (1904), 207-267, 459-533.

[31] G. N. Watson, Theory of Bessel Functions, 2nd ed., University Press, Cambridge, 1966.

Department of Mathematics, University of Illinois, 1409 West Green Street, Urbana, IL 61801, USA

E-mail address: berndt@math.uiuc.edu

Department of Mathematics and Statistics, Dalhousie University, Halifax, Nova Scotia, B3H 3J5 CANADA

E-mail address: math@oyeat.com

Department of Mathematics, University of Illinois, 1409 West Green Street, Urbana, IL 61801, USA

E-mail address: sglim@uiuc.edu

Department of Mathematics, University of Illinois, 1409 West Green Street, Urbana, IL 61801, USA

E-mail address: zaharesc@math.uiuc.edu 